\title{
LOCALLY UNIFORM SPACES
}

\author{
BY \\ JAMES WILLIAMS
}

\begin{abstract}
The axioms for a locally uniform space $(X, \mathscr{U})$ may be obtained by localizing the last axiom for a uniform space to obtain $\forall x \in X, \forall U \in \mathscr{U}, \exists V \in \mathscr{V}:(V \circ V)[x]$ $\subseteq U[x]$. With each locally uniform space one may associate a regular topology, just as one associates a completely regular topology with each uniform space. The topologies of locally uniform spaces with nested bases may be characterized using Boolean algebras of regular open sets. As a special case, one has that locally uniform spaces with countable bases have pseudo-metrizable topologies.

Several types of Cauchy filters may be defined for locally uniform spaces, and a major portion of the paper is devoted to a study and comparison of their properties. For each given type of Cauchy filter, complete spaces are those in which every Cauchy filter converges; to complete a space is to embed it as a dense subspace in a complete space. In discussing these concepts, it is convenient to make the mild restriction of considering only those locally uniform spaces $(X, \mathscr{V})$ in which each element of $\mathscr{V}$ is a neighborhood of the diagonal in $X \times X$ with respect to the relative topology; these spaces I have called NLU-spaces.

With respect to the more general types of Cauchy filters, some NLU-spaces are not completable; this happens even though some completable NLU-spaces can still have topologies which are not completely regular. Examples illustrating these completeness situations and having various topological properties are obtained from a generalized construction. It is also shown that there is a largest class of Cauchy filters with respect to which each NLU-space has a completion that is also an NLU-space.
\end{abstract}

Preface. For any locally uniform space $(X, \mathscr{V})$, the sets of the form $V[x]$, for $x \in X$ and $V \in \mathscr{V}$, describe a neighborhood system for a regular topological space. Conversely, the neighborhood system for any regular space $X$ is generated by the local uniformity consisting of all neighborhoods of the diagonal in $X \times X$.

If a local uniformity has a nested base, then the generated topology is paracompact. From this it follows directly that a local uniformity with a countable base has a pseudo-metrizable topology. On the other hand, if the topology of a regular space has a $\sigma$-locally finite base, it is easy to construct from such a base, a local uniformity with a countable base which generates this topology, thereby demonstrating Nagata's result that a regular $T_{1}$-space whose topology has a $\sigma$-locally finite base is metrizable. In fact the condition needed for the construction is somewhat weaker than $\sigma$-local finiteness.

Using a localized form of écart space, Niemytski has proved a result which says essentially that local uniformities with countable bases have pseudo-metrizable

Received by the editors February 10, 1970 and, in revised form, September 1, 1971. AMS 1969 subject classifications. Primary 5410, 5430; Secondary 5425, 5435, 5440, 5450. Copyright (C) 1972, American Mathematical Society 
topologies; it is contained in a 1927 paper to which I was led by a series of accidents after completing my own work. In view of the fact that Nagata's result follows so easily from Niemytski's, it is surprising that the two results were separated by a long period; and the connection does not seem to have been pointed out since.

1. Introductory concepts. When not otherwise specified, the terminology here will be like that in Kelley [9]. It will be convenient to develop our ideas about locally uniform spaces in terms of the more general class of semi-uniform spaces, whose theory is presented in Čech [2]. A semi-uniformity on a set $X$ is a filter $\mathscr{V}$ on $X \times X$ such that, for each $V \in \mathscr{V}$,

(1) $\Delta(X)=\{\langle x, x\rangle: x \in X\} \subseteq V$ and

(2) $V^{-1}=\{\langle y, x\rangle:\langle x, y\rangle \in V\} \in \mathscr{V}$.

If $\mathscr{V}$ is a semi-uniformity on a set $X$, then a base (subbase) for $\mathscr{V}$ is just a base (subbase) for $\mathscr{V}$ considered as a filter; the pair $(X, \mathscr{V})$ is a semi-uniform space; the elements of $\mathscr{V}$ are called semineighborhoods of the diagonal in $X \times X$; and for each $x \in X, \mathscr{N}_{x}=\{V[x]: V \in \mathscr{V}\}$ is the semineighborhood system at $x$ generated by $\mathscr{V} ;$ in case the family $\left\{\mathscr{N}_{x}: x \in X\right\}$ is a neighborhood system for a topology $\mathscr{T}$ on $X$, we say that $\mathscr{T}$ is the topology generated by $\mathscr{V}$. We shall call those semi-uniformities which have generated topologies topological.

1.1 Theorem. Let $(X, \mathscr{V})$ be a topological semi-uniform space. Then for each $A \subseteq X$ and $M \subseteq X \times X$, we have

$$
A^{-}=\bigcap\{V[A]: V \in \mathscr{V}\} \text { and } M^{-}=\bigcap\{V \circ M \circ V: V \in \mathscr{V}\} \text {, }
$$

where $A^{-}$and $M^{-}$are the closures of $A$ and $M$ in $X$ and $X \times X$ respectively, with respect to the generated topology of $\mathscr{V}$.

Proof. This is essentially Theorem 6.6 of Kelley [9].

Definition. We shall say that a subbase $\mathscr{B}$ for a semi-uniformity on a set $X$ is locally uniform iff $\forall U \in \mathscr{B}, \forall x \in X, \exists V \in \mathscr{V}:(V \circ V)[x] \subseteq U[x]$. If $\mathscr{V}$ is a locally uniform semi-uniformity, we shall call $\mathscr{V}$ a local uniformity, and call $(X, \mathscr{V})$ a locally uniform space.

Definition. For any set $X$ and any $V \subseteq X \times X$, we let $V^{2}=V \circ V$, and for each $n \in \omega-\{0\}$, let $V^{n+1}=V^{n} \circ V$.

1.2 TheOREM. If $(X, \mathscr{V})$ is a locally uniform space, then $\forall n \in \omega-\{0\}, \forall U \in \mathscr{V}$, $\forall x \in X, \exists V \in \mathscr{V}: V^{n}[x] \subseteq U[x]$.

Proof. This follows directly from the above definition.

1.3 LemMA. Every locally uniform space is topological.

Proof. Let $(X, \mathscr{V})$ be a locally uniform space; pick $x \in X$. The collection of all semineighborhoods of $x$ is a filter since $\mathscr{V}$ is. Pick $U \in \mathscr{V}$ and let $(U[x])^{\circ}$ $=\{y \in X: \exists V \in \mathscr{V} ; V[y] \subseteq U[x]\}$. Clearly $x \in(U[x])^{\circ} \subseteq U[x]$, and it remains to show that $(U[x])^{\circ}$ is a semineighborhood of each of its points. For any given 
$y \in(U[x])^{\circ}$, pick $V \in \mathscr{V}$ so that $V[y] \subseteq U[x]$; then choosing $W \in \mathscr{V}$ so that $W[W(y)]=W^{2}[y] \subseteq V[y] \subseteq U[x]$ shows that $W[y] \subseteq(U[x])^{\circ}$; hence $(U[x])^{\circ}$ is a semineighborhood of $y$.

The converse of the above lemma is, incidentally, false; a topological space $X$ may be generated by a semi-uniform space if and only if $\forall x, y \in X, x \in\{y\}^{-}$iff $y \in\{x\}^{-}$, by Theorem 23B.3, p. 402 of Čech [2].

The following theorem is contained in the unpublished work of G. D. Richardson; similar theorems relating regular topological spaces to other generalizations of uniform spaces have also been given by A. S. Davis [4, Theorem 4], S. A. Naimpally [12, Theorem 2], and K. Morita [10, Part I, Theorem 1, and Part IV in his discussion of "Uniformities" which "agree strongly with the topology]."

1.4 THEOREM. The topology of a locally uniform space is regular. Conversely, for any regular space $X$, the set of all neighborhoods of the diagonal in $X \times X$ is a local uniformity which generates the topology of $X$.

Proof. Suppose $(X, \mathscr{V})$ is a locally uniform space. For any given $x \in X$ and neighborhood $V[x]$, with $V \in \mathscr{V}$, if $U \in \mathscr{V}$ is such that $U^{2}[x] \subseteq V[x]$, then $(U[x])^{-}$ $\subseteq U[U[x]] \subseteq V[x]$ by Theorem 1.1. Hence each neighborhood of each point has a closed subneighborhood.

Now suppose $X$ is a regular topological space and let $\mathscr{V}$ be the set of all neighborhoods of the diagonal in $X \times X$. It is clear that $\mathscr{V}$ is a semi-uniformity, and for given $U \in \mathscr{V}$ and $x \in X$, if $A, B$, and $C$ are open neighborhoods of $x$ such that $C^{-} \subseteq B \subseteq B^{-} \subseteq A \subseteq U[x]$, then $V=(B \times B) \cup\left[\left(A-C^{-}\right) \times\left(A-C^{-}\right)\right] \cup\left[\left(X-B^{-}\right) \times\right.$ $\left.\left(X-B^{-}\right)\right]$is an open neighborhood of the diagonal such that

$$
V^{2}[x]=V[B]=A \subseteq U[x] .
$$

Definition. For any set $K$, we shall abbreviate the product $K \times K$ to $K^{\times 2}$. While this notation for Cartesian squares is only necessary for very long expressions, it increases the readability of most; for consistency, we shall use it throughout.

Definition. We shall say that a subbase $\mathscr{B}$ for a semi-uniformity $\mathscr{V}$ on a set $X$ has the neighborhood property iff $\forall U \in \mathscr{B}, \forall x \in X, \exists V \in \mathscr{V}:(V[x])^{\times 2} \subseteq U$. If $\mathscr{V}$ is a local uniformity with the neighborhood property, we shall call $\mathscr{V}$ an $N L U$ or neighborhood local uniformity, and call $(X, \mathscr{V})$ an $N L U$-space.

The following simple results are useful for identifying local uniformities and NLU's. The almost trivial proofs of $1.5,1.6$, and 1.9 have been omitted.

1.5 THEOREM. A subbase $\mathscr{B}$ for a topological semi-uniformity has the neighborhood property iff each $V \in \mathscr{B}$ is a neighborhood of the diagonal in $X^{\times 2}$.

1.6 THEOREM. A semi-uniformity $\mathscr{V}$ on a set $X$ is an NLU iff it has the property that $\forall U \in \mathscr{V}, \forall x \in X, \exists V \in \mathscr{V}:\left(V^{2}[x]\right)^{\times 2} \subseteq U$.

1.7 THEOREM. Let $X$ be a regular topological space; suppose $\mathscr{B}$ is a collection of symmetric neighborhoods of the diagonal in $X^{\times 2}$ such that for each neighborhood $M$ 
of $x$, there is a neighborhood $N$ of $x$ and an element $V \in \mathscr{B}$ such that $V[N] \subseteq M$. Then $\mathscr{B}$ is a subbase for an NLU generating the topology of $X$.

Proof. Let $\mathscr{B}$ and $X$ satisfy the above condition. $\mathscr{B}$ is clearly a subbase for a semi-uniformity. The condition shows as a special case that $\mathscr{B}$ generates the topology of $X$. Hence $\mathscr{B}$ has the neighborhood property by Theorem 1.5. It remains to show that $\mathscr{B}$ has the local uniform property. Given $x \in X$ and $U \in \mathscr{B}$, we may choose $V \in \mathscr{B}$ and a neighborhood $B$ of $x$ so that $V[B] \subseteq U[x]$, then we may choose $W \in \mathscr{B}$ so that $W[x] \subseteq B$, so that $(W \cap V)^{2}[x] \subseteq V[W[x]] \subseteq V[B] \subseteq U[x]$. Consequently $\mathscr{B}$ is a subbase for an NLU generating the topology of $X$.

Definition. If $\mathscr{U}$ and $\mathscr{V}$ are semi-uniformities on a set $X, \mathscr{U}$ is stronger than $\mathscr{V}$ iff $\mathscr{U} \supseteq \mathscr{V}$.

1.8 THEOREM. If $\mathscr{U}$ is a local uniformity on a set $X$, then there is a strongest NLU $\mathscr{V}$ contained in $\mathscr{U} . \mathscr{U}$ and $\mathscr{V}$ have the same topologies.

Proof. Let $\mathscr{U}$ be a local uniformity on a set $X$, let $\mathscr{W}$ be the set of all neighborhoods of the diagonal in $X^{\times 2}$. We shall show that $\mathscr{U} \cap \mathscr{W}$ is the required structure: $\mathscr{U} \cap \mathscr{W}$ is clearly the strongest structure we might consider, and it has the filter and symmetry properties to make it a semi-uniformity. It generates the topology of $(X, \mathscr{U})$ since for each $x \in X$ and neighborhood $U[x]$, with $U \in \mathscr{U}$, we may choose $W \in \mathscr{W}$ so that $W[x]=U[x]$ by Theorem 1.4 ; so that $(U \cup W)[x]$ $=U[x]$, with $U \cup W \in \mathscr{U} \cap \mathscr{W} . \mathscr{U} \cap \mathscr{W}$ has the neighborhood property by Theorem 1.5 and we show the local uniform property as follows: Pick $x \in X$ and $N \in \mathscr{U} \cap \mathscr{W}$, choose $W \in \mathscr{W}$ so that $W^{2}[x] \subseteq N[x]$ and $U \in \mathscr{U}$ so that $U^{2}[x] \subseteq N[x]$ and $U[x] \subseteq W[x]$, and $\hat{W} \in \mathscr{W}$ so that $\hat{W}[x] \subseteq U[x]$ and $\hat{W} \subseteq W$, then

$$
\begin{aligned}
(\hat{W} \cup U)^{2}[x] & =\left(\hat{W}^{2} \cup(\hat{W} \circ U) \cup(U \circ \hat{W}) \cup U^{2}\right)[x] \\
& \subseteq W^{2}[x] \cup U^{2}[x] \subseteq N[x],
\end{aligned}
$$

with $\hat{W} \cup U \in \mathscr{U} \cap \mathscr{W}$.

1.9 THEOREM. Suppose $\mathscr{V}$ is a local uniformity on a set $X$ and $\mathscr{U}$ is a topological semi-uniformity on $X$ which is stronger than $\mathscr{V}$ and has the same topology, then $\mathscr{U}$ is a local uniformity.

Definition. If $\mathscr{V}$ is a local uniformity, then for each $n \in \omega-\{0\}$, let

$$
\mathscr{V}^{n}=\left\{U \subseteq X^{\times 2}: \exists V \in \mathscr{V}: V^{n} \subseteq U\right\} .
$$

1.10 TheOREM. For each local uniformity $\mathscr{V}$ and each $n \in \omega-\{0,1\}, \mathscr{V}^{n}$ is an $N L U$ with the same topology as $\mathscr{V}$.

Proof. Let $\mathscr{V}$ be a local uniformity on a set $X$; it suffices to prove the theorem for $\mathscr{V}^{2}$. First, $\mathscr{V}^{2}$ is clearly a semi-uniformity. Pick $W \in \mathscr{V}^{2}$, pick $x \in X$, and choose $V \in \mathscr{V}$ so that $V^{2} \subseteq W$; choose $U \in \mathscr{V}$ so that $U^{4}[x] \subseteq V[x]$, then $U^{2} \in \mathscr{V}^{2}$ and $\left(\left(U^{2}\right)^{2}[x]\right)^{\times 2} \subseteq(V[x])^{\times 2} \subseteq W$, so that $\mathscr{V}^{2}$ is a neighborhood local uniformity by 
Theorem 1.6. The relative topology of $\mathscr{V}^{2}$ is of course weaker than that of $\mathscr{V}$, and it is also stronger since $\mathscr{V}$ is locally uniform.

Two other properties which one might require of a local uniformity are that it have a base of open sets, or that it have a base of closed sets. I do not know whether it is reasonable to make both requirements in the case where the topology is not completely regular, but one can, however, say the following:

1.11 TheOREM. If $(X, \mathscr{V})$ is an NLU-space and $\mathscr{B}=\left\{V^{\circ}: V \in \mathscr{V}\right\}$, then $\mathscr{B}$ is an open base for an $N L U \mathscr{U}$ which is stronger than $\mathscr{V}$ and generates the same topology; $\mathscr{U}$ is of course the weakest such structure having these properties.

Proof. Let $(X, \mathscr{V})$ and $\mathscr{B}$ be as above. $\mathscr{B}$ is obviously a base for a semi-uniformity $\mathscr{U}$ which is stronger than $\mathscr{V}$ and generates the topology of $X$. $\mathscr{U}$ has the neighborhood property by Theorem 1.5 and is locally uniform by Theorem 1.9.

1.12 THEOREM. If $(X, \mathscr{V})$ is a locally uniform space and $\mathscr{B}=\left\{V^{-}: V \in \mathscr{V}\right\}$, then $\mathscr{B}$ is a closed base for a local uniformity $\mathscr{U}$ which is weaker than $\mathscr{V}$ and has the same topology; $\mathscr{U}$ is of course the strongest such local uniformity having these properties.

Proof. Let $(X, \mathscr{V})$ and $\mathscr{B}$ be as above. $\mathscr{B}$ is obviously a base for a semi-uniformity $\mathscr{U}$ which is weaker than $\mathscr{V}$; by Theorem 1.1 it is stronger than $\mathscr{V}^{3}$. By Theorem 1.10 it must generate the same topology as $\mathscr{V}$ and $\mathscr{V}^{3}$. So by Theorem 1.9 $\mathscr{U}$ is a local uniformity.

Finally, we shall consider some methods for comparing locally uniform spaces; they will be used later for studying completeness.

Definition. Two local uniformities $\mathscr{V}$ and $\mathscr{W}$ are weakly equivalent if for some $n, m \in \omega-\{0\}, \mathscr{V}^{n} \subseteq \mathscr{W}$ and $\mathscr{W}^{m} \subseteq \mathscr{V}$.

. A local uniformity $\mathscr{V}$ is of course weakly equivalent to each $\mathscr{V}^{n}$. Moreover, using the fact that for each $V \in \mathscr{V},\left(V^{3}\right)^{\circ}$ is an open neighborhood of $V$, it is easy to see that $\mathscr{V}$ is weakly equivalent to a local uniformity with an open base, and also weakly equivalent to one with a closed base. Two weakly equivalent local uniformities generate the same topology by Theorem 1.10, and they tend to give the same kind of information about the space; it is thus natural to consider as a uniformlike structure for a regular space, equivalence classes of locally uniform spaces; in which case the uniform spaces consist precisely of those equivalence classes which contain only one element. Concepts defined in terms of such equivalence classes suggest corresponding statements about the local uniformities belonging to them, and we shall apply the terms "weak" and "strong" to such statements, according to what is appropriate.

Definition. Suppose $(X, \mathscr{U})$ and $(Y, \mathscr{V})$ are locally uniform spaces, a function $f:(X, \mathscr{U}) \rightarrow(Y, \mathscr{V})$ is uniform iff $\forall V \in \mathscr{V}, \exists U \in \mathscr{U}: \tilde{f}[U] \subseteq V$ (where $\tilde{f}(x, y)=$ $\langle f(x), f(y)\rangle)$. $f$ is weakly uniform iff for some $n \in \omega-\{0\}, f:(X, \mathscr{U}) \rightarrow\left(Y, \mathscr{V}^{n}\right)$ is uniform. $f$ is a (weakly) uniform isomorphism iff $f$ and $f^{-1}$ are one-to-one and onto (weakly) uniform functions. 
With respect to these definitions, it is easy to see that the composition of (weakly) uniform functions is (weakly) uniform, and that two local uniformities $\mathscr{U}$ and $\mathscr{V}$ on a set $X$ are equal (weakly equivalent) iff the identity $\iota:(X, \mathscr{U}) \rightarrow(X, \mathscr{V})$ is a uniform (weakly uniform) isomorphism.

1.13 THEOREM. (Weakly) uniform functions on locally uniform spaces are continuous with respect to the relative topologies.

Proof. Let $(X, \mathscr{U})$ and $(Y, \mathscr{V})$ be locally uniform spaces; it suffices to show that if for given $n \in \omega-\{0\}, f:(X, \mathscr{U}) \rightarrow\left(Y, \mathscr{V}^{n}\right)$ is uniform, then $f$ is continuous. Pick $x \in X$; for each neighborhood $N$ of $f(x)$ in $Y$, we may choose $V \in \mathscr{V}$ and $U \in \mathscr{U}$ so that $V^{n}[f(x)] \subseteq N$ and $\tilde{f}[U] \subseteq V^{n}$, so that

$$
f[U[x]]=\tilde{f}[U][f(x)] \subseteq V^{n}[f(x)] \subseteq N .
$$

Hence $f$ is continuous.

If $(Y, \mathscr{V})$ is a locally uniform space and $X \subseteq Y$, it need not be the case that $\mathscr{V}^{2} \mid X=(\mathscr{V} \mid X)^{2}$; in fact the two local uniformities need not be weakly equivalent, this being the case in Example 3.4; hence we make the following:

Definition. A locally uniform space $(Y, \mathscr{V})$ is a strong extension of a subspace $(X, \mathscr{V} \mid X)$ iff $\forall n \in \omega-\{0\}, \mathscr{V}^{n} \mid X$ and $(\mathscr{V} \mid X)^{n}$ are weakly equivalent.

1.14 THEOREM. $(Y, \mathscr{V})$ is a strong extension of $(X, \mathscr{U})$ iff $\forall n \in \omega-\{0\}, \exists m \in \omega-\{0\}$, $\forall U \in \mathscr{U}, \exists V \in \mathscr{V}: U^{m} \supseteq V^{n} \cap X^{\times 2}$.

Proof. This follows directly from the fact that for each $n \in \omega-\{0\}, \mathscr{V}^{n} \mid X$ must be weaker than $(\mathscr{V} \mid X)^{n}=\mathscr{T}^{n}$.

2. Local uniformities with nested bases. Our first main task will be to show that a locally uniform space $(X, \mathscr{V})$ with a nested base has a paracompact topology. The strategy will be to show first that each neighborhood of the diagonal in $X^{\times 2}$ is uniformizable, and next to show that $\mathscr{V}$ may be replaced by a uniformity with a nested base which generates the same topology. Paracompactness then follows, in the pseudo-metrizable case by A. H. Stone's paracompactness theorem, and in the non-pseudo-metrizable case from a result by E. Zakon. The nearly trivial proofs of the following two lemmas are omitted.

2.1 Lemma. If a local uniformity has a nested base, it has a nested symmetric base.

2.2 LEMmA. If $\mathscr{B}$ is a nested base for a local uniformity $\mathscr{V}$, then $\left\{V^{2}: V \in \mathscr{B}\right\}$ is a nested base for the $N L U \mathscr{V}^{2}$.

The proof of the following theorem uses a technique introduced by Niemytski [13, p. 511].

2.3 THEOREM. If a local uniformity $\mathscr{U}$ on a set $X$ has a nested base, then the set of all neighborhoods of the diagonal in $X^{\times 2}$ is a uniformity. 
Proof. By the above lemma, we may assume that $\mathscr{U}$ is an NLU. It suffices to show that for each symmetric neighborhood $V$ of the diagonal there is a neighborhood $U$ of the diagonal such that $U \circ U \subseteq V$; so choose $V$ to be any symmetric neighborhood of the diagonal. By Lemma $2.1, \mathscr{U}$ has a symmetric nested base $\mathscr{A}$. Let $\mathscr{B}=\{V \cap U: U \in \mathscr{A}\} . \mathscr{B}$ is a nested collection of symmetric neighborhoods of the diagonal. $\mathscr{B}$ generates the topology of $\mathscr{U}$, and is a base for a semi-uniformity stronger than $\mathscr{U}$. Therefore by Theorems 1.5 and $1.9, \mathscr{B}$ is a base for an NLU $\mathscr{V}$.

Since $V$ is an element of $\mathscr{V}, \forall x \in X, \exists W \in \mathscr{V}:(W[x])^{\times 2} \subseteq V$; and $\exists U \in \mathscr{V}$ : $U^{3}[x] \subseteq W[x]$, so that $\left(U^{3}[x]\right)^{\times 2} \subseteq V$. Thus $\forall x \in X$, we may choose $U_{x} \in \mathscr{B}$ in such a way that $\left(U_{x}^{3}[x]\right)^{\times 2} \subseteq V$. Let $U=\bigcup\left\{\left(U_{x}[x]\right)^{\times 2}: x \in X\right\}$. $U$ is a symmetric neighborhood of the diagonal.

Claim. $U \circ U \subseteq V$. Pick $x, y, z \in X$ so that $\langle x, y\rangle,\langle y, z\rangle \in U$, then there exist points $r, s \in X$ such that $x, y \in U_{r}[r]$ and $y, z \in U_{s}[s]$. Since $U_{r}, U_{s} \in \mathscr{B}$, one is a subset of the other. Suppose for instance that $U_{r} \subseteq U_{s}$. By assumption $z \in U_{s}[s]$. On the other hand, using the symmetry of $U_{r}$ we have $x \in U_{r}[r], r \in U_{r}[y]$, and $y \in U_{s}[s]$, so that $x \in U_{r}^{2}\left[U_{s}[s]\right] \subseteq U_{s}^{3}[s]$. Thus

$$
\langle x, z\rangle \in U_{s}[s] \times U_{s}^{3}[s] \subseteq U_{s}^{3}[s] \times U_{s}^{3}[s] \subseteq V .
$$

Therefore $U \circ U \subseteq V$.

Definition. For any semi-uniformity $\mathscr{U}$, we shall define the cofinality of $\mathscr{U}$ to be the least cardinal $\kappa$ for which $\mathscr{U}$ has a base of cardinality $\kappa$.

2.4 THEOREM. Suppose that $(X, \mathscr{U})$ is a topological semi-uniform space, and that $\mathscr{U}$ has a nested base and has cofinality $\kappa$. Then $\kappa$ has the property that for each collection $\mathscr{C}$ of open subsets of $X$ with cardinality less than $\kappa, \cap \mathscr{C}$ is open. If $X$ has a nonisolated point, then $\kappa$ is the largest cardinal with this property. Also, for any collection $\mathscr{P}$ of neighborhoods of the diagonal in $X^{\times 2}$ of cardinality less than $\kappa, \cap \mathscr{P}$ is a neighborhood of the diagonal.

Proof. The first statement differs little from Proposition 26, p. 133 of Isbell [8]. To prove the second, suppose that $x$ is a nonisolated point of $X$, then the neighborhood system at $x$ has a nested open base of cardinality $\kappa$, and its intersection $\bar{x}$ is not open. This shows the maximality of $\kappa$. Finally, let $\mathscr{P}$ be a collection of neighborhoods of the diagonal in $X^{\times 2}$ of cardinality less than $\kappa$. Pick $x \in X$; for each $P \in \mathscr{P}$ there is a neighborhood $N_{P}$ of $x$ such that $N_{P}^{\times 2} \subseteq P$. Let $K=\bigcap\left\{N_{p}: P \in \mathscr{P}\right\}$, then $K$ must be a neighborhood of $x$ and $K^{\times 2} \subseteq \bigcap \mathscr{P}$. Hence $\bigcap \mathscr{P}$ is a neighborhood of the diagonal.

2.5 THEOREM. An NLU with a nested base may be extended to a uniformity with a nested base, which has the same topology and (at most) the same cofinality.

Proof. Let $\mathscr{V}$ be an NLU with a nested base and with cofinality $\kappa$, defined on a set $X$. Let $\left\{V_{\alpha}: \alpha \in \kappa\right\}$ be a base for $\mathscr{V}$. Let $U_{0}=V_{0} \cap V_{0}^{-1}$. We proceed by induction for each $\lambda \in \kappa$ to choose symmetric neighborhoods $U_{\lambda}$ of the diagonal in $X^{\times 2}$. If 
$\lambda \in \kappa$ and $U_{\lambda}$ has been chosen, then by Theorem 2.3, there is a symmetric neighborhood $U_{\lambda+1}$ of the diagonal in $X^{\times 2}$ such that $U_{\lambda+1} \subseteq V_{\lambda+1}$ and $U_{\lambda+1} \circ U_{\lambda+1} \subseteq U_{\lambda}$. If $\lambda$ is a limit ordinal less than $\kappa$ and each $U_{\alpha}$ for $\alpha<\lambda$ has been chosen, let $U_{\lambda}=\bigcap\left\{U_{\alpha}: \alpha<\lambda\right\} ; U_{\lambda}$ is a neighborhood of the diagonal by the above theorem. By construction, $\left\{U_{\lambda}: \lambda \in \kappa\right\}$ is a nested base for a uniformity generating the topology of $(X, \mathscr{V})$.

2.6 COROLlARy. A local uniformity with a countable base has a pseudo-metrizable topology.

Proof. This follows directly from the above and Theorem 6.13 of Kelley [9].

Theorems similar to the above corollary have been given by Niemytski [13], A. H. Frink [5, Theorem 4], J. Suzuki [15, Theorem 1], and K. Morita [10, §IV, Theorem 4]. The nice proof of the following result was suggested by the referee, who noticed that, in view of the above theorems, most of E. Zakon's results on uniformities with quasi-nested bases [16] carry over to local uniformities with nested bases.

2.7 THEOREM. Let $(X, \mathscr{U})$ be a (locally) uniform space; suppose $\mathscr{U}$ has a nested base and is not pseudo-metrizable; then every open cover of $X$ has a discrete refinement.

Proof. By Lemma 2.2 and Theorem 2.5 is suffices to prove the result for the case where $(X, \mathscr{U})$ is a uniform space. By Theorem 2.1d of [16], we may let $\left\{V_{\alpha}: \alpha \in \kappa\right\}$ be a family of equivalence relations on $X$ which generate the topology of $X$, and are nested in such a way that $V_{\alpha} \subseteq V_{\beta}$ iff $\beta \geqq \alpha$. Let $\mathscr{A}$ be any open cover of $X$. Let $\mathscr{B}$ be the set of all $V_{\lambda}[x]$ for which $x \in X$ and $\lambda$ is the first ordinal such that $\exists A \in \mathscr{A}: V_{\lambda}[x] \subseteq A . \mathscr{B}$ clearly refines $\mathscr{A}$ and covers $X$. To see that $\mathscr{B}$ is discrete, suppose for example that for $\lambda \leqq \eta<\kappa, V_{\lambda}[x], V_{\eta}[y] \in \mathscr{B}$, and $V_{\lambda}[x] \cap V_{\eta}[y] \neq \varnothing$. Using the fact that $\left\{V_{\alpha}: \alpha<\kappa\right\}$ is a nested family of equivalence relations, we see that $V_{\lambda}[y] \cap V_{\lambda}[x] \neq \varnothing$, so that $V_{\lambda}[x]=V_{\lambda}[y] \supseteq V_{\eta}[y]$. Then $\eta=\lambda$ because both are the first ordinal $\alpha$ such that $V_{\alpha}[y]$ is contained in some element of $\mathscr{A}$. Hence $V_{\eta}[y]=V_{\lambda}[x]$.

2.8 COROLlaRY. Every (locally) uniform space with a nested base has a paracompact topology.

Proof. This follows immediately from Stone's paracompactness theorem for pseudo-metric spaces [14] and the above result.

We now show how to construct local uniformities with nested bases for a certain class of topological spaces:

2.9 TheOREM. Let $(X, \mathscr{T})$ be a regular topological space, suppose $\left\{\mathscr{A}_{\alpha}: \alpha \in \kappa\right\}$ is a nested collection of families such that

(1) $\bigcup\left\{\mathscr{A}_{\alpha}: \alpha \in \kappa\right\}$ is a base for $\mathscr{T}$,

(2) $\forall \alpha \in \kappa, \forall \mathscr{B} \subseteq \mathscr{A}_{\alpha}, \cap \mathscr{B}$ and $\cap\{X-\bar{B}: B \in \mathscr{B}\}$ are open.

Then $\mathscr{T}$ is generated by a local uniformity with a nested base indexed by $\kappa$. 
Proof. Let $(X, \mathscr{T})$ and $\left\{\mathscr{A}_{\alpha}: \alpha \in \kappa\right\}$ be as above. We may assume without loss of generality that each $\mathscr{A}_{\alpha}$ contains $X$ and the empty set. Pick $\alpha \in \kappa$; for each $x \in X$,

let $M_{x}^{\alpha}=\bigcap\left\{A: x \in A\right.$ and $\left.A \in \mathscr{A}_{\alpha}\right\}$,

let $N_{x}^{\alpha}=\bigcap\left\{X-A^{-}: x \in X-A^{-}\right.$and $\left.A \in \mathscr{A}_{\alpha}\right\}$,

let $V_{\alpha}=\bigcup\left\{\left(M_{x}^{\alpha} \cap N_{x}^{\alpha}\right)^{\times 2}: x \in X\right\}$.

Claim. $\left\{V_{\alpha}: \alpha \in \kappa\right\}$ is a nested base for an NLU generating the topology of $X$. It is nested since $\forall \alpha, \beta \in \kappa$, if $\mathscr{A}_{\alpha} \subseteq \mathscr{A}_{\beta}$, then $V_{\alpha} \supseteq V_{\beta}$. Each $V_{\alpha}$ is symmetric by construction, and from our assumption about $\mathscr{A}_{\alpha}$, each $M_{x}^{\alpha} \cap N_{x}^{\alpha}$ is an open neighborhood of $x$, so that $V_{\alpha}$ is a neighborhood of the diagonal in $X^{\times 2}$ as well. In order to show that $\left\{V_{\alpha}: \alpha \in \kappa\right\}$ is a base for an NLU generating the topology of $X$, it is thus sufficient by Theorem 1.7 to show that $\forall x \in X, \forall$ neighborhood $A$ of $x, \exists V_{\alpha}, \exists$ neighborhood $B$ of $x: V_{\alpha}[B] \subseteq A$. Pick $x \in X$ and $A \in \bigcup \mathscr{A}_{\alpha}$, then we may choose $\beta \in \kappa$, and $B \in \mathscr{A}_{\beta}$, so that $x \in B \subseteq \bar{B} \subseteq A$ and $A \in \mathscr{A}_{\beta}$; in which case

$$
V_{\alpha}[B]=\bigcup\left\{M_{y}^{\alpha} \cap N_{y}^{\alpha}: M_{y}^{\alpha} \cap N_{y}^{\alpha} \cap B \neq \varnothing, y \in X\right\} .
$$

Now if $y \in A$, then $M_{y}^{\alpha} \subseteq A$, if $y \notin A$, then $y \notin \bar{B}$ and $N_{y}^{\alpha} \cap B=\varnothing$. Hence

$$
V_{\alpha}[B] \subseteq \bigcup\left\{M_{y}^{\alpha} \cap N_{y}^{\alpha}: y \in A\right\}=A .
$$

If in the above theorem each $\mathscr{A}_{\alpha}$ is locally finite, then the space $(X, \mathscr{T})$ will be pseudo-metrizable, as we will soon show. In any case, if the index set $\kappa$ is countable, we have immediately that $(X, \mathscr{T})$ is pseudo-metrizable by Corollary 2.6. For any locally finite family $\mathscr{A}$, and any subfamily $\mathscr{B}$ of $\mathscr{A}$, it is easy to see that $\bigcap \mathscr{B}$ is open and that $\bigcup\{\bar{B}: B \in \mathscr{B}\}$ is closed, so that $\bigcap\{X-\bar{B}: B \in \mathscr{B}\}$ is also open. Consequently, the above theorem implies Nagata's metrization theorem [11] that a regular topological space with a $\sigma$-locally finite base is pseudo-metrizable.

2.10 Proposition. Suppose that $X$ is a regular topological space and that $\left\{\mathscr{A}_{\alpha}: \alpha \in \Gamma\right\}$ is a nested collection of locally finite families whose union is a base for $X$. Then $X$ is pseudo-metrizable.

Proof. By Theorem $2.9 X$ has a local uniformity $\mathscr{U}$ with a nested base. Let $\kappa$ be the cofinality of $\mathscr{U}$. If $\kappa=\aleph_{0}$, then $X$ is pseudo-metrizable by Corollary 2.6. If for each $x \in X,\{\bar{x}\}$ is open, then $X$ is pseudo-metrizable by the pseudo-metric $d$ given by $d(x, y)=0$ if $x \in\{y\}^{-}$, and $d(x, y)=1$ otherwise. So assume $\kappa>\boldsymbol{N}_{0}$, and that $x$ is a point of $X$ for which $\{\bar{x}\}$ is not open, we shall derive a contradiction. Let $\left\{\mathscr{A}_{\alpha_{n}}: n \in \omega\right\}$ be a countable subcollection of $\left\{\mathscr{A}_{\alpha}: \alpha \in \Gamma\right\}$ such that for each $n \in \omega, \mathscr{A}_{\alpha_{n}}$ contains a neighborhood of $x$ and $\mathscr{A}_{\alpha_{n+1}}$ contains a neighborhood of $x$ which is strictly smaller than any neighborhood of $x$ in $\mathscr{A}_{\alpha_{n}}$. Let

$$
N=\bigcap\left\{A \in \mathscr{A}_{\alpha_{n}}: x \in A \text { and } n \in \omega\right\} .
$$

$N$ is the countable intersection of open sets and since $\kappa>\boldsymbol{\aleph}_{0}, N$ is an open neighborhood of $x$. Let $\mathscr{A}_{\beta}$ be a family in $\left\{\mathscr{A}_{\alpha}: \alpha \in \Gamma\right\}$ which contains a subneighborhood 
$B$ of $N$. $B$ does not belong to any $\mathscr{A}_{\alpha_{n}}$, hence $\forall n \in \omega, \mathscr{A}_{\beta} \supseteq \mathscr{A}_{\alpha_{n}}$. But then $\forall n \in \omega$, $\exists A \in \mathscr{A}_{\alpha_{n+1}}-\mathscr{A}_{\alpha_{n}}: x \in A \in \mathscr{A}_{\beta}$; so that every neighborhood of $x$ meets infinitely many elements of $\mathscr{A}_{\beta}$, a contradiction.

Theorem 2.9 gives a sufficient condition for the existence of a local uniformity with a nested base on a topological space. The condition is given in terms of two latticelike properties of families in a nested collection.

We shall now consider in more detail the relation between lattice properties of open sets and local uniformities with nested bases. In the process we will derive a converse for Theorem 2.9.

Definition. An open subset of a topological space is regular iff it is equal to the interior of its closure.

For any subset $A$ of a topological space $X, A^{-\circ}$ is a regular open set. Consequently, if the topology of $X$ is regular and has a base $\mathscr{B}$, then $\left\{B^{-\circ}: B \in \mathscr{B}\right\}$ is a base of regular open subsets of $X$. In any case, the family of all regular open subsets of $X$ is a complete Boolean algebra, where the operations of meet, join, and complement are given by $\bigwedge \mathscr{A}=(\cap \mathscr{A})^{-\circ}, \bigvee \mathscr{A}=(\bigcup \mathscr{A})^{-\circ}$, and $\sim A=X-A^{-}$. This result is presented in Halmos [6].

Definition. By a regular decomposition of a topological space $X$ we shall mean a pairwise disjoint family $\mathscr{A}$ of regular open sets such that $\bigcup \mathscr{A}$ is dense in $X$.

2.11 LEMMA. If the intersection of a family of regular open sets is open, then it is regular.

Proof. Let $\mathscr{P}$ be a family of regular open sets whose intersection is open; we need to show that $\bigcap \mathscr{P}=(\bigcap \mathscr{P})^{-\circ}$. First $\bigcap \mathscr{P}=(\cap \mathscr{P})^{\circ} \subseteq(\cap \mathscr{P})^{-\circ}$. On the other hand, for each $P \in \mathscr{P},(\bigcap \mathscr{P})^{-\circ} \subseteq P^{-\circ}=P$, so that $(\bigcap \mathscr{P})^{-\circ} \subseteq \bigcap \mathscr{P}$.

2.12 Lemma. Every regular decomposition $\mathscr{A}$ of a topological space $X$ is an atomic generating set for a complete subalgebra $\mathscr{B}$ of the Boolean algebra $\mathscr{R}$ of all regular open subsets of $X$. If $\mathscr{A}$ is locally finite, then the meet operation in $\mathscr{B}$ is just the set-intersection operation.

Proof. Let $\mathscr{A}, \mathscr{R}$, and $X$ be as above, and let $\bigvee$ and $\sim$ be the join and complement operations in $\mathscr{R}$. Let $\mathscr{B}=\{\bigvee \mathscr{P}: \mathscr{P} \subseteq \mathscr{A}\}$. We shall show that $\mathscr{B}$ is a complete subalgebra of $\mathscr{R}$.

(1) $\forall \mathscr{P} \subseteq \mathscr{A}, \sim \bigvee \mathscr{P}=\bigvee(\mathscr{A}-\mathscr{P})$ : Since $X-(\bigcup \mathscr{P})^{-0-}=X-(\bigcup \mathscr{P})^{-}$, it suffices to show $(\bigcup(\mathscr{A}-\mathscr{P}))^{-\circ}=X-(\bigcup \mathscr{P})^{-}$. Since $\bigcup \mathscr{A}$ is dense, we have

$$
\begin{aligned}
X & =(\bigcup \mathscr{A})^{-}=(\bigcup \mathscr{P})^{-} \cup(\bigcup(\mathscr{A}-\mathscr{P}))^{-} \\
& =(\bigcup \mathscr{P})^{-} \cup(\bigcup(\mathscr{A}-\mathscr{P}))^{-\circ} ;
\end{aligned}
$$

so $X-(\bigcup \mathscr{P})^{-} \subseteq(\bigcup(\mathscr{A}-\mathscr{P}))^{-\circ}$. On the other hand, $\bigcup(\mathscr{A}-\mathscr{P}) \subseteq X-\bigcup \mathscr{P}$, so $\bigcup(\mathscr{A}-\mathscr{P}) \subseteq(X-\bigcup \mathscr{P})^{\circ}=X-(\bigcup \mathscr{P})^{-}$, and so $(\bigcup(\mathscr{A}-\mathscr{P}))^{-\circ} \subseteq\left(X-(\bigcup \mathscr{P})^{-}\right)^{-\circ}$ $=X-(\bigcup \mathscr{P})^{-}$. 
(2) If $\left\{\mathscr{P}_{\alpha}: \alpha \in \Gamma\right\}$ is any given collection of subfamilies of $\mathscr{A}$, then

$$
\bigvee\left\{\bigvee \mathscr{P}_{\alpha}: \alpha \in \Gamma\right\}=\bigvee \bigcup\left\{\mathscr{P}_{\alpha}: \alpha \in \Gamma\right\} \in \mathscr{B}:
$$

First,

$$
\begin{aligned}
\bigvee\left\{\bigvee \mathscr{P}_{\alpha}: \alpha \in \Gamma\right\} & =\left(\bigcup\left\{\left(\bigcup \mathscr{P}_{\alpha}\right)^{-\circ}: \alpha \in \Gamma\right\}\right)^{-\circ} \\
& \supseteq\left(\bigcup\left\{\bigcup \mathscr{P}_{\alpha}: \alpha \in \Gamma\right\}\right)^{-\circ}=\bigvee \bigcup\left\{\mathscr{P}_{\alpha}: \alpha \in \Gamma\right\} .
\end{aligned}
$$

But also, $\left(\bigcup\left\{\left(\bigcup \mathscr{P}_{\alpha}\right)^{-}: \alpha \in \Gamma\right\}\right)^{-}=\left(\bigcup\left\{\bigcup \mathscr{P}_{\alpha}: \alpha \in \Gamma\right\}\right)^{-}$, so that

$$
\begin{aligned}
\bigvee\left\{\bigvee \mathscr{P}_{\alpha}: \alpha \in \Gamma\right\} & \subseteq\left(\bigcup\left\{\left(\bigcup \mathscr{P}_{\alpha}\right)^{-}: \alpha \in \Gamma\right\}\right)^{-\circ} \\
& =\left(\bigcup\left\{\bigcup \mathscr{P}_{\alpha}: \alpha \in \Gamma\right\}\right)^{-\circ} \\
& =\bigvee \bigcup\left\{\mathscr{P}_{\alpha}: \alpha \in \Gamma\right\} .
\end{aligned}
$$

(3) Suppose $\mathscr{A}$ is locally finite, then the meet operation in $\mathscr{B}$ coincides with setintersection: It suffices to show that the intersection of a collection of elements of $\mathscr{B}$ is open, and therefore regular, by the previous lemma. But this will follow directly from the fact that for each $x \in X$, there is a smallest element of $\mathscr{B}$ containing $x$. Given $x \in X$, let $\mathscr{A}_{x}=\left\{A \in \mathscr{A}: x \in A^{-}\right\}$. Since $\mathscr{A}$ is locally finite, we have $x \notin \bigcup\left\{A^{-}: A \in \mathscr{A}-\mathscr{A}_{x}\right\}=\left(\bigcup\left(\mathscr{A}-\mathscr{A}_{x}\right)\right)^{-}$. Hence, using (1), we have

$$
x \in X-\left(\cup\left(\mathscr{A}-\mathscr{A}_{x}\right)\right)^{-}=\sim\left(\bigvee\left(\mathscr{A}-\mathscr{A}_{x}\right)\right)=\bigvee \mathscr{A}_{x}
$$

Clearly, $\mathscr{A}_{x}$ is the smallest collection for which $x \in \bigvee \mathscr{A}_{x}$.

2.13 LemMA. Let $\mathscr{C}$ be any locally finite cover of a topological space $X$ by regular open sets. Then $\mathscr{C}$ generates a complete atomic subalgebra $\mathscr{B}$ of the algebra of all regular open subsets of $X$; the atoms of $\mathscr{B}$ form a locally finite regular decomposition of $X$.

Proof As before, we let $\mathscr{R}$ be the algebra of all regular open subsets of $X$, and $\forall A \in \mathscr{R}$, let $\sim A=X-A^{-}$. Let $\Gamma$ be the set of all functions $\phi$ from $\mathscr{C}$ to $\mathscr{R}$ such that $\forall A \in \mathscr{C}$, either $\phi(A)=A$ or $\phi(A)=\sim A$. Let $\mathscr{A}$ be the collection of all nonempty sets of the form $\bigcap\{\phi(A): A \in \mathscr{C}\}$, with $\phi \in \Gamma$. We shall show that $\mathscr{A}$ is a locally finite regular decomposition of $X$.

(1) Each given element $A$ of $\mathscr{A}$ is open and therefore regular: Suppose $x \in A \in \mathscr{A}$. Using the local finiteness of $\mathscr{C}$, we see easily that there is a neighborhood $N$ of $x$ which meets only those elements of $\mathscr{C}$ whose closures contain $x$. From the definition of $A$, it follows that $N \subseteq A$. Hence $A$ is open.

(2) Each point of $X$ belongs to the closure of some element of $\mathscr{A}$, so that $\bigcup \mathscr{A}$ is dense in $X$ : First we prove the following fact. If $\mathscr{P}$ is a finite subcollection of $\mathscr{C}$ and $x \in \bigcap\left\{P^{-}: P \in \mathscr{P}\right\}$, then for some $\phi \in \Gamma, x \in(\bigcap\{\phi(P): P \in \mathscr{P}\})^{-}$. Proof: if $\mathscr{P}$ has one element, the fact is trivial. Suppose we have shown the fact for $n$ elements, and suppose $\mathscr{P}$ has $n+1$ elements, pick $P \in \mathscr{P}$. We choose $\phi \in \Gamma$ so that

$$
x \in(\bigcap\{\phi(R): R \in \mathscr{P}-\{P\}\})^{-} ;
$$


let $Q=\bigcap\{\phi(R): R \in \mathscr{P}-\{P\}\}$. Claim: either $x \in(Q \cap P)^{-}$or $x \in(Q \cap \sim P)^{-}$, since otherwise there is an open neighborhood $N$ of $x$ such that $N \cap Q \cap P=\varnothing$ and $N \cap Q \cap(X-P)^{-}=\varnothing$, so that $N \cap Q$ is a nonempty open subset of $(X-P)$ $\cap P^{-}$, which is the boundary of $P$, a contradiction. Hence we may choose $\phi^{\prime} \in \Gamma$ so that $x \in\left(Q \cap \phi^{\prime}(P)\right)^{-}$and $\forall R \in \mathscr{P}-\{P\}, \phi(R)=\phi^{\prime}(R)$, so that

$$
x \in\left(\bigcap\left\{\phi^{\prime}(P): P \in \mathscr{P}\right\}\right)^{-} .
$$

If $\mathscr{C}_{x}=\left\{A \in \mathscr{C}: x \in A^{-}\right\}$, then we may use the above fact to choose $\phi \in \Gamma$ so that $x \in\left(\bigcap\left\{\phi(A): A \in \mathscr{C}_{x}\right\}\right)^{-}$, and may further require of $\phi$ that $\forall A \in \mathscr{C}-\mathscr{C}_{x}, \phi(A)=$ $\sim A$, so that $\bigcap\left\{\phi(A): A \in \mathscr{C}-\mathscr{C}_{x}\right\}$ is a neighborhood of $x$ by local finiteness. From this we conclude that $x \in(\bigcap\{\phi(A): A \in \mathscr{C}\})^{-}$.

(3) The local finiteness of $\mathscr{C}$ clearly carries over to $\mathscr{A}$, and $\mathscr{A}$ is pairwise disjoint by construction. This together with steps (1) and (2) shows that $\mathscr{A}$ is a locally finite regular decomposition of $X$. Hence by the previous lemma, there is a complete subalgebra $\mathscr{B}$ of $\mathscr{P}$ for which $\mathscr{A}$ is an atomic generating set. From the construction of $\mathscr{A}$ it is clear that any complete subalgebra containing $\mathscr{C}$ contains $\mathscr{A}$ also. Thus we have left to prove only that $\mathscr{C} \subseteq \mathscr{B}$. Claim: for each given $C \in \mathscr{C}$,

$$
C=(\bigcup\{A \in \mathscr{A}: A \subseteq C\})^{-\circ}=\bigvee\{A \in \mathscr{A}: A \subseteq C\} \in \mathscr{B} .
$$

Clearly $(\bigcup\{A \in \mathscr{A}: A \subseteq C\})^{-\circ} \subseteq C^{-\circ}=C$; on the other hand, if $x \in C$ and

$$
\mathscr{A}_{x}=\left\{A \in \mathscr{A}: x \in A^{-}\right\},
$$

then each $A \in \mathscr{A}_{x}$ meets $C$, and must therefore be a subset of $C$, but then $\left(\cup \mathscr{A}_{x}\right)^{-\circ}$ is a neighborhood of $x$ contained in $\bigvee\{A \in \mathscr{A}: A \subseteq \mathscr{C}\}$.

2.14 TheOREM. A regular topological space $X$ has a (local) uniformity with a nested base iff its topology has a base which is the union of a nested collection of complete atomic Boolean algebras of regular open sets whose meet operations coincide with the set-intersection operation.

Proof. First suppose $X$ is a regular topological space with a base which is the union of a nest collection of complete atomic Boolean algebras whose meet operations are set-intersections. This collection clearly satisfies the hypotheses of Theorem 2.9, and so $X$ has a local uniformity with a nested base.

Now suppose $X$ has a local uniformity with a nested base. We shall first consider the case in which $X$ is pseudo-metrizable. Let $\left\{\mathscr{C}_{n}^{\prime}: n \in \omega\right\}$ be a nested collection of locally finite open covers of $X$ whose union is a base for the topology of $X . \forall n \in \omega$, let $\mathscr{C}_{n}=\left\{C^{-\circ}: C \in \mathscr{C}_{n}^{\prime}\right\}$; then $\left\{\mathscr{C}_{n}: n \in \omega\right\}$ is a nested collection of locally finite families of regular open sets and its union is a base for the topology of $X$. By the above lemma, each $\mathscr{C}_{n}$ generates a complete atomic Boolean algebra $\mathscr{B}_{n}$. Thus $\left\{\mathscr{B}_{n}: n \in \omega\right\}$ is the required nested family.

We next suppose that $X$ is not pseudo-metrizable. Let $\mathscr{U}$ be a (local) uniformity for $X$ with a nested base. By Lemma 2.2 and Theorem 2.5, it suffices to assume $\mathscr{U}$ 
is a uniformity. By Theorem 2.1d of Zakon [16], $\mathscr{U}$ has a nested base $\left\{V_{\alpha}: \alpha \in \kappa\right\}$ consisting of equivalence relations. For each $\alpha$, let $\mathscr{A}_{\alpha}=\left\{V_{\alpha}[x]: x \in X\right\}$. Then each $\mathscr{A}_{\alpha}$ is a discrete family of clopen sets, and the collection $\left\{\mathscr{A}_{\alpha}: \alpha \in \kappa\right\}$ is linearly ordered by refinement. For each $\alpha \in \kappa$, let $\mathscr{B}_{\alpha}=\left\{\bigcup \mathscr{P}: \mathscr{P} \subseteq \mathscr{A}_{\alpha}\right\}$. Each $\mathscr{B}_{\alpha}$ is, with respect to intersection, union, and set complementation in $X$, a complete algebra of clopen (and therefore regular open) sets. Hence $\left\{\mathscr{B}_{\alpha}: \alpha \in \kappa\right\}$ is the required nested collection.

2.15 COROLlary. A regular topological space $X$ has a (local) uniformity with a nested base iff there is a refinement-nested collection of regular locally finite decompositions of $X$ whose union is a basis for the algebra of all regular open subsets of $X$.

Proof. In view of the above theorem it suffices to mention that if for each $\alpha \in \kappa$, $\mathscr{A}_{\alpha}$ is an atomic basis for the complete algebras $\mathscr{B}_{\alpha}$, and if $\bigcup\left\{\mathscr{B}_{\alpha}: \alpha \in \kappa\right\}$ is a base for the topology of $X$, then $\bigcup\left\{\mathscr{B}_{\alpha}: \alpha \in \kappa\right\}$ and hence $\bigcup\left\{\mathscr{A}_{\alpha}: \alpha \in \kappa\right\}$ is a basis for the algebra of all regular open subsets of $X$.

3. Completeness. The possibilities for both "weak" and "strong" comparisons which were given at the end of $\S 1$ also arise with Cauchy filters:

Definition. For each $n \in \omega-\{0\}$, a weak Cauchy filter of degree $n$ on a semiuniform space $(X, \mathscr{V})$ is a filter $\mathscr{F}$ on $X$ such that $\forall V \in \mathscr{V}, \exists F \in \mathscr{F}: F \times F \subseteq V^{n}$. A Cauchy filter is a weak Cauchy filter of degree 1. A locally uniform space is (strongly) complete iff every (weak) Cauchy filter on $X$ converges with respect to the relative topology of $\mathscr{V}$; a (strong) completion of $(X, \mathscr{V})$ is a (strongly) complete locally uniform space $(Y, \mathscr{W})$ containing $(X, \mathscr{V})$ as a dense subspace.

While the above definitions seem reasonable by comparison with the similar concepts in uniform spaces, they do not represent the only possibilities, and study of their failings will help motivate the additional Cauchy ideas which we will consider in $\$ 6$.

3.1 THEOREM. If $(X, \mathscr{U})$ and $(Y, \mathscr{V})$ are weakly uniformly isomorphic locally uniform spaces, then $(X, \mathscr{U})$ is strongly complete iff $(Y, \mathscr{V})$ is. In particular, if $\mathscr{U}$ and $\mathscr{V}$ are weakly equivalent structures on a set $X$, then $(X, \mathscr{U})$ is strongly complete iff $(X, \mathscr{V})$ is.

Proof. Let $f:(X, \mathscr{U}) \rightarrow(Y, \mathscr{V})$ be a weakly uniform isomorphism of locally uniform spaces and suppose for example that $(Y, \mathscr{V})$ is strongly complete, then for some $n \in \omega-\{0\} ; \forall V \in \mathscr{U}, \exists U \in \mathscr{U}: \tilde{f}[U] \subseteq V^{n}$. If $\mathscr{F}$ is a weak Cauchy filter of degree $m$ on $(X, \mathscr{U})$, then for each $V \in \mathscr{V}$, we may choose $U \in \mathscr{U}$ so that $\tilde{f}[U] \subseteq V^{n}$ and choose $F \in \mathscr{F}$ so that $F \times F \subseteq U^{m}$, so that $\tilde{f}[F \times F] \subseteq\left(V^{n}\right)^{m}=V^{n m}$. Thus $f[\mathscr{F}]$ is a base for a weak Cauchy filter of degree $n m$ on $(Y, \mathscr{V})$, and hence converges; hence $\mathscr{F}$ itself converges since $f$ is a homeomorphism, by Theorem 1.13.

As a result of the following theorem, we shall often consider completeness problems only for NLU-spaces. 
3.2 THEOREM. In a semi-uniform space with the neighborhood property, convergent filters are Cauchy. If a weak Cauchy filter on a locally uniform space has adherent $\left({ }^{1}\right)$ points, it concerges to each of them.

Proof. Suppose the filter $\mathscr{F}$ converges to a point $x$ in the neighborhood semiuniform space $\left(X, \mathscr{V}^{\circ}\right)$. Then for each $V \in \mathscr{V}$, we may choose $U \in \mathscr{V}$ and $F \in \mathscr{F}$ so that $(U[x])^{\times 2} \subseteq V$ and $F \subseteq U[x]$, so that $F \times F \subseteq V$.

If $\mathscr{F}$ is a weak Cauchy filter of degree $n$ on the locally uniform space $(X, \mathscr{V})$ and $x$ is an adherent point of $\mathscr{F}$, then for any neighborhood $A$ of $x$, we may choose $V \in \mathscr{V}$ so that $V^{n+1}[x] \subseteq A$ and $F \in \mathscr{F}$ so that $F \times F \subseteq V^{n}$. Since $x$ is an adherent point, some $y \in X$ belongs to $V[x] \cap F$; consequently $F \subseteq V^{n}[y] \subseteq V^{n+1}[x] \subseteq A$.

Suppose $(X, \mathscr{I})$ is a subspace of $(Y, \mathscr{V})$ and $\mathscr{F}$ is a filter on $X$. If $\mathscr{F}$ is weak Cauchy on $(X, \mathscr{O})$, then it is weak Cauchy in $(Y, \mathscr{V})$ since necessarily $\forall V \in \mathscr{V}$, $\forall n \in \omega-\{0\}, V^{n} \cap X^{\times 2} \supseteq\left(V \cap X^{\times 2}\right)^{n}$. However, if $\mathscr{F}$ is weak Cauchy in $(Y, \mathscr{V})$ it need not be weak Cauchy in $(X, \mathscr{U})$, a fact which will often be a nuisance.

3.3 THEOREM. If a local uniform space $\left(Y, \mathscr{V}^{*}\right)$ is a strong extension of $(X, \mathscr{U})$, and $\mathscr{F}$ is a filter on $X$ which is weak Cauchy in $\left(Y, \mathscr{Y}^{\prime}\right)$, then $\mathscr{F}$ is weak Cauchy on $(X, \mathscr{U})$.

Proof. Let $(X, \mathscr{I}),\left(Y, \mathscr{Y}^{*}\right)$, and $\mathscr{F}$ be as above. Suppose $\mathscr{F}$ is weak Cauchy of degree $n$ in $\left(Y, \mathscr{Y}^{\circ}\right)$ and suppose $m \in \omega-\{0\}$ is such that for each given $U \in \mathscr{U}$, $\exists V \in \mathscr{V}: U^{m} \supseteq V^{n} \cap X^{\times 2}$; if we then choose $F \in \mathscr{F}$ so that $F \times F \subseteq V^{n}$, we will also have $F \times F \subseteq U^{m}$. Hence $\mathscr{F}$ is weak Cauchy of degree $m$ on $(X, \mathscr{U})$.

3.4 Example of a "bad" extension: Let $\boldsymbol{R}$ be the set of real numbers; we define a base for a local uniformity $\mathscr{V}^{\prime}$ on $Y=\boldsymbol{R} \times\{0,1\}$ as follows: $\forall n \in \omega-\{0\}$, let

$$
V_{n}=\left\{\langle x, y\rangle \in X^{\times 2}:\left|x_{1}-y_{1}\right|<1 / n \text { and } x_{2}=y_{2} \text {, or } x_{1}, y_{1}>n \text { and } x_{2} \neq y_{2}\right\} \text {. }
$$

The collection $\left\{V_{n}: n \in \omega-\{0\}\right\}$ clearly generates the expected product topology on $\boldsymbol{R} \times\{0,1\}$, and using Theorem 1.7, it is easy to see that it is a base for an NLU as well. Let $X=\boldsymbol{R} \times\{0\}$, and let $\mathscr{F}=\left\{F \subseteq X: \exists n \in \omega: F \supseteq\left\{x \in X: x_{1}>n\right\}\right\}$, then $\mathscr{F}$ is weak Cauchy in $Y$ of degree 2 , but is not weak Cauchy on $X$. Also, $\mathscr{V}^{2} \mid X$ is not weakly equivalent to $(\mathscr{V} \mid X)^{2}$.

Finally, we shall give some simple compactness theorems for local uniform spaces.

3.5 ThEOREM $\left({ }^{2}\right)$. Let $(X, \mathscr{V})$ be a strongly complete $N L U$-space, then a closed subset $A$ of $X$ is compact iff every infinite subset of $A$ has a limit point in $A$.

Proof. Suppose $(X, \mathscr{V})$ is strongly complete, $A$ is a closed subset of $X$, and every infinite subset of $A$ has a limit point, but $A$ is not compact. We may let $\mathscr{F}$ be an ultrafilter on $A$ which does not converge. If it were the case that $\forall U \in \mathscr{V}, \exists x_{U} \in A$ : $U\left[x_{U}\right] \in \mathscr{F}$, then $\mathscr{F}$ would be weak Cauchy of degree 2 in $(X, \mathscr{V})$. So we may choose

(1) The set of all adherent points of a filter $\mathscr{F}$ on a topological space $X$ is $\cap\left\{F^{-}: F \in \mathscr{F}\right\}$.

$\left({ }^{2}\right)$ I suspect this theorem holds for complete spaces as well. 
a symmetric $U \in \mathscr{V}$ such that $\forall x \in A, U[x] \notin \mathscr{F}$. Pick $x_{0} \in A$, and for each $n \geqq 1$, pick $x_{n} \in\left(A-\bigcup_{j=0}^{n-1} U\left[x_{j}\right]\right) \in \mathscr{F}$. Then the set $\left\{x_{j}: j \in \omega\right\}$ has a limit point $y$ in $A$. Let $V \in \mathscr{V}$ be such that $(V[y])^{\times 2} \subseteq U$, then for infinitely many distinct $x_{i}$ and $x_{j}$, $\left\langle x_{i}, x_{j}\right\rangle \in(V[y])^{\times 2} \subseteq U$, contrary to the choice of the $x_{n}$ 's.

3.6 LEMMA. If $X$ is a compact topological space and $V$ is a closed subset of $X^{\times 2}$, then $V^{2}$ is also closed.

Proof. Let $X$ and $V$ be as above. If $\langle x, z\rangle \in\left(V^{2}\right)^{-}$, then for any neighborhoods $N$ of $x$ and $M$ of $z, N \times M \cap V^{2} \neq \varnothing$ and so $V[N] \cap V^{-1}[M] \neq \varnothing$; the collection of all such sets $V[N] \cap V^{-1}[M]$ is a filter base, which must have an adherent point $y$, by compactness. For any neighborhoods $N$ of $x, K$ of $y$, and $M$ of $z$, we must have $K \cap\left(V[N] \cap V^{-1}[M]\right) \neq \varnothing$, so that $(N \times K) \cap V \neq \varnothing$ and $(K \times M)$ $\cap V \neq \varnothing$. Hence $\langle x, y\rangle$ and $\langle y, z\rangle$ belong to $V^{-}=V$, so $\langle x, z\rangle \in V^{2}$. Hence $V^{2}$ is closed.

3.7 THEOREM. If $X$ is a regular compact topological space, then there is a unique $N L U$ which generates the topology of $X$.

Proof. Let $X$ be a regular compact space. Since there is only one uniformity generating the topology of $X$, it suffices to show that if $\mathscr{V}$ is any given NLU generating the topology of $X$, then $\mathscr{V}$ is a uniformity: By Theorem 1.12, the closed elements of $\mathscr{V}$ form a base for a local uniformity generating the topology of $X$, so that for each given $U \in \mathscr{V}$, and each $x \in X$, we may let $V_{x}$ be a closed element of $\mathscr{V}$ such that $V_{x}^{2}[x] \subseteq U^{\circ}[x]$. Then $\forall y \in X$,

$$
\left(\bigcap\left\{V_{x}^{2}: x \in X\right\}\right)[y] \subseteq V_{y}^{2}[y] \subseteq U^{\circ}[y] .
$$

Hence $\bigcap\left\{V_{x}^{2}: x \in X\right\} \subseteq U^{\circ}$. Each $V_{x}^{2}$ is closed and $X^{\times 2}$ is compact, hence the intersection of a finite number of the sets $V_{x}^{2}$ is contained in the open set $U^{\circ}$; we have

$$
U \supseteq \bigcap_{j=1}^{n} V_{x_{j}}^{2} \supseteq\left(\bigcap_{j=1}^{n} V_{x_{j}}\right)^{2},
$$

with $\bigcap_{j=1}^{n} V_{x_{j}} \in \mathscr{V}$. Hence $\mathscr{V}$ is a uniformity.

4. Piecing together a locally finite collection. We shall now consider the following type of problem: Suppose $\left\{C_{\alpha}\right\}$ is a locally finite cover of a topological space $X$, and for each $\alpha, \mathscr{V}_{\alpha}$ is a local uniformity generating the topology of $C_{\alpha}$. Is $X$ locally uniformizable, and under what conditions is there a local uniformity $\mathscr{V}$ such that each $\mathscr{V}_{\alpha}$ is equal to $\mathscr{V} \mid C_{\alpha}$ ? As a special case we will get Nagata's piecing together theorem for metric spaces. By way of comparison, we shall consider in addition the problem of piecing together a locally finite collection of uniform spaces.

4.1 Lemma. Suppose we have the following things:

(1) $X$, a topological space;

(2) $\mathscr{C}$, a locally finite closed cover of $X$; 
(3) for each $C \in \mathscr{C} ; \mathscr{V}_{C}$, a local uniformity on $C$;

(4) $\Gamma$, a set of choice functions from $\mathscr{C}$ to $\bigcup\left\{\mathscr{V}_{C}: C \in \mathscr{C}\right\}$ such that

(i) $\forall C \in \mathscr{C}, \Gamma[C]$ is a symmetric base for $\mathscr{V}_{C}$;

(ii) $\forall f, g \in \Gamma, f(\cap) g \in \Gamma$, where $\forall C \in \mathscr{C} ;(f(\cap) g)(C)=f(C) \cap g(C)$.

For each $f \in \Gamma$, let $U_{f}=\bigcup f[\mathscr{C}]$. Then $\left\{U_{f}: f \in \Gamma\right\}$ is a symmetric base for a local uniformity which generates the topology of $X$.

Proof. Assume hypotheses (1) through (4): $\left\{U_{f}: f \in \Gamma\right\}$ is a symmetric base for a semi-uniformity since it is clear that $\forall f, g \in \Gamma ; \Delta \subseteq U_{f}, U_{f} \cap U_{g}=U_{f(\cap) g}$ and $U_{f}$ is symmetric (since for each $C \in \mathscr{C}, f(C)$ is).

To show that $\left\{U_{f}: f \in \Gamma\right\}$ generates the topology of $X$, pick $x \in X$, and let $C_{1}, \ldots, C_{n}$ be the elements of $\mathscr{C}$ which contain $x$; first, every neighborhood of $x$ is generated by $\left\{U_{f}: f \in \Gamma\right\}$ : Let $N$ be any subneighborhood of $\bigcup_{j=1}^{n} C_{j}$. For each $j=1, \ldots, n$, pick $V_{j} \in \mathscr{V}_{C_{j}}$ so that $V_{j}[x] \subseteq N \cap C_{j}$. By making repeated use of the fact that $\forall g, h \in \Gamma, g(\cap) h \in \Gamma$; we may pick $f \in \Gamma$ so as to satisfy the finite number of conditions $f\left(C_{j}\right) \subseteq V_{j}, j=1, \ldots, n$. Then $N \supseteq \bigcup_{j=1}^{n} V_{j}[x] \supseteq \bigcup_{j=1}^{n} f\left(C_{j}\right)[x]=U_{f}[x]$. Hence $\left\{U_{f}: f \in \Gamma\right\}$ generates every neighborhood of $x$. Second, we show that $\left\{U_{f}: f \in \Gamma\right\}$ generates only neighborhoods of $x$ : Pick $g \in \Gamma$; for each $k=1, \ldots, n$; let $P_{k}$ be a neighborhood of $x$ such that $P_{k} \cap C_{k} \subseteq g\left(C_{k}\right)[x]$ and $P_{k} \subseteq \bigcup_{j=1}^{n} C_{j}$. Then

$$
U_{g}[x]=\bigcup_{j=1}^{n} g\left(C_{j}\right)[x] \supseteq \bigcup_{j=1}^{n}\left(\bigcap_{k=1}^{n} P_{k}\right) \cap C_{j}=\bigcap_{k=1}^{n} P_{k} .
$$

Finally, we show that $\left\{U_{f}: f \in \Gamma\right\}$ has the local uniform property; it suffices to show that $\forall x \in X, \forall$ neighborhood $K$ of $x, \exists f \in \Gamma: U_{f}^{2}[x] \subseteq K$. To this end, we assemble the following list, given $x$ in $X$ and any neighborhood $K$ of $x$.

(i) $C_{1}, \ldots, C_{n}$ the elements of $\mathscr{C}$ containing $x$.

(ii) For each $j=1, \ldots, n ; V_{j}$, an element of $\mathscr{V}_{C_{j}}$ such that $V_{j}^{2}[x] \subseteq K \cap C_{j}$.

(iii) $N$, a subneighborhood of $\bigcup_{j=1}^{n} C_{j}$ such that $\forall j=1, \ldots, n ; N \cap C_{j} \subseteq V_{j}[x]$.

(iv) $f$, an element of $\Gamma$ such that $\forall j=1, \ldots, n ; f\left(C_{j}\right) \subseteq V_{j}$ and $f\left(C_{j}\right)[x] \subseteq N$.

Now, $U_{f}[x]=\bigcup_{j=1}^{n} f\left(C_{j}\right)[x] \subseteq N \subseteq \bigcup_{j=1}^{n} C_{j}$. So that

$$
\begin{aligned}
U_{f}\left[U_{f}[x]\right] & =\bigcup_{j=1}^{n} f\left(C_{j}\right)\left[U_{f}[x]\right] \\
& \subseteq \bigcup_{j=1}^{n} f\left(C_{j}\right)[N]=\bigcup_{j=1}^{n} f\left(C_{j}\right)\left[N \cap C_{j}\right] \\
& \subseteq \bigcup_{j=1}^{n} V_{j}\left[V_{j}[x]\right] \subseteq K \cap\left(\bigcup_{j=1}^{n} C_{j}\right) .
\end{aligned}
$$

4.2 COROLLARY. If a topological space has a locally finite closed cover of regular subspaces, then it has a regular topology.

Proof. Suppose a space $X$ has such a cover $\mathscr{C}$. For each $C \in \mathscr{C}$, let $\mathscr{V}_{C}$ be the set of all neighborhoods of the diagonal in $C$. Let $\Gamma$ be all choice functions $f$ from $\mathscr{C}$ to $\bigcup\left\{\mathscr{V}_{C}: C \in \mathscr{C}\right\}$ such that $\forall C \in \mathscr{C}, f(C)$ is symmetric; clearly $\Gamma$ satisfies conditions (i) and (ii) in the above lemma. Hence $X$ is local-uniformizable, and therefore regular. 
4.3 CoRollary $\left({ }^{3}\right)$. If $\mathscr{C}$ is a locally finite closed cover of a topological space $X$ by pseudo-metrizable subspaces, then $X$ is pseudo-metrizable.

Proof. In the hypotheses for Lemma 4.1, we let each $\mathscr{V}_{C}$ be a pseudo-metrizable uniformity and let $\left\{V_{C_{n}}: n \in \omega\right\}$ be a nested symmetric base for $\mathscr{V}_{C}$. For elements of $\Gamma$, we choose the functions $f_{n}$ defined by $\forall C \in \mathscr{C}, f_{n}(C)=V_{C_{n}}$. $\Gamma$ has properties (i) and (ii) since $\forall C \in \mathscr{C}, \Gamma[C]=\left\{V_{C_{n}}: n \in \omega\right\}$ and $\forall f_{n}, f_{m} \in \Gamma, f_{n}(\cap) f_{m}=f_{\max \{n, m\}}$. We conclude that the sets $U_{n}=\bigcup f_{n}[\mathscr{C}]$ form a countable base for a local uniformity generating the topology of $X$. Hence $X$ is pseudo-metrizable by Corollary 2.6.

In Lemma 4.1 we constructed a local uniformity $\mathscr{V}$ such that for each of the given subspaces $C \in \mathscr{C}, \mathscr{V} \mid C$ and $\mathscr{V}_{C}$ had the same topology; however, in order to construct $\mathscr{V}$ so that $\mathscr{V} \mid C=\mathscr{V}_{C}$, it is necessary and sufficient that $\mathscr{C}$ be a compatible collection in the following sense:

Definition. A collection $\Theta$ of locally uniform spaces is compatible iff for any two elements $(X, \mathscr{U})$ and $(Y, \mathscr{V})$ of $\theta, \mathscr{U}|Y=\mathscr{V}| X ; \Theta$ is strongly compatible iff $\forall n \in \omega-\{0\},\left\{\left(X, \mathscr{V}^{n}\right):(X, \mathscr{V}) \in \Theta\right\}$ is compatible.

Definition. A family $\mathscr{C}$ of subsets of a set is star finite iff each $C \in \mathscr{C}$ meets only finitely many elements of $\mathscr{C}$.

4.4 THEOREM. Let $X$ be a topological space, $\mathscr{C}$ a locally finite closed cover for $X$, and $\left\{\left(C, \mathscr{V}_{C}\right): C \in \mathscr{C}\right\}$ a compatible collection of (neighborhood) locally uniform spaces. Then

(I) there is a strongest (neighborhood) local uniformity $\mathscr{U}$ such that $\forall C \in \mathscr{C}$, $\mathscr{U} \mid C=\mathscr{V}_{C} . \mathscr{U}$ generates the topology of $X$.

(II) If, in addition, $\mathscr{C}$ is star finite, each $\mathscr{V}_{C}$ is a strongly complete $N L U$, and $\left\{\left(C, \mathscr{V}_{C}\right): C \in \mathscr{C}\right\}$ is strongly compatible, then $\mathscr{U}$ is strongly complete.

Proof of I. In the hypotheses for Lemma 4.1, we take $\Gamma$ to be the set of all choice functions $f: \mathscr{C} \rightarrow \bigcup\left\{\mathscr{V}_{C}: C \in \mathscr{C}\right\}$ such that $\forall C \in \mathscr{C}, f(C)$ is symmetric. $\Gamma$ satisfies conditions (i) and (ii); hence the collection of all sets of the form $U_{f}=f[\mathscr{C}]$ is a base for a local uniformity $\mathscr{U}$ generating the topology of $X$.

(1) $\mathscr{U}$ is the strongest local uniformity such that $\forall C \in \mathscr{C}, \mathscr{U} \mid C=\mathscr{V}_{C}$ : Pick $C \in \mathscr{C}$. First, $\mathscr{U} \mid C \subseteq \mathscr{V}_{C}$ since $\forall g \in \Gamma, U_{g} \cap(C \times C) \supseteq g(C) \in \mathscr{V}_{C}$. Second, $\mathscr{U} \mid C \supseteq \mathscr{V}_{C}$ : Pick $V_{C} \in \mathscr{V}_{C}$; for each $D \in \mathscr{C}$, we may, by compatibility, choose $V_{D} \in \mathscr{V}_{D}$ so that $V_{D} \cap C \times C=V_{C} \cap D \times D$. Define $g \in \Gamma$ by $\forall D \in \mathscr{C}, g(D)=V_{D}$. Then $U_{g} \cap(C \times C)$ $=V_{C}$. Third, if $\mathscr{W}$ is any local uniformity such that $\forall C \in \mathscr{W}, \mathscr{W} \mid C=\mathscr{V}_{C}$, then $\mathscr{W} \subseteq \mathscr{U}$ : For each symmetric $W \in \mathscr{W}$, let $g \in \Gamma$ be given by $g(C)=W \cap(C \times C)$; then $U_{g} \subseteq W$. Hence $\mathscr{W} \subseteq \mathscr{U}$.

(2) Assume now that each $\mathscr{V}_{C}$ is an NLU; we shall show that there is a strongest NLU $\mathscr{U}^{\prime}$ such that $\forall C \in \mathscr{C}, \mathscr{U}^{\prime} \mid C=\mathscr{V}_{C}$ : Let $\mathscr{U}^{\prime}$ be the set of all neighborhoods of the diagonal which belong to $\mathscr{U}$. By Theorem $1.8, \mathscr{U}^{\prime}$ is the strongest NLU

( $\left.{ }^{3}\right)$ This result is Nagata's Theorem 2 of [11], stated for pseudo-metric spaces. 
contained in $\mathscr{U}$; so by the properties of $\mathscr{U}$ given in step (1), we have $\forall C \in \mathscr{C} ; \mathscr{U}^{\prime} \mid C \subseteq$ $\mathscr{V}_{C}$; and if $\mathscr{U}^{\prime} \mid C \supseteq \mathscr{V}_{C}$, then $\mathscr{U}^{\prime}$ is the strongest NLU with these properties. To show for any given $C \in \mathscr{C}$, that $\mathscr{U}^{\prime} \mid C \supseteq \mathscr{V}_{C}$, let $V$ be any symmetric element of $\mathscr{V}_{C}$; choose $f \in \Gamma$ so that $U_{f} \cap C \times C=V$; let $W^{\prime}$ be an $X \times X$-neighborhood of $\Delta(C)$, the diagonal in $C \times C$, such that $W^{\prime} \cap C \times C=V$; let $W=W^{\prime} \cup(X-C)^{\times 2}$. Then, since $C$ is closed in $X, W$ is a neighborhood of the diagonal in $X \times X$ such that $W \cap$ $(C \times C)=V$; so then $W \cup U_{f} \in \mathscr{U}^{\prime}$ and $\left(W \cup U_{f}\right) \cap C \times C=V$. Hence $\mathscr{U}^{\prime} \mid C \supseteq \mathscr{V}_{C}$.

Proof of II. Let $\mathscr{\mathscr { I }}$ and $\mathscr{U}^{\prime}$ be as above. Assume that $\mathscr{C}$ is star finite, that each $\mathscr{V}_{C}$ is strongly complete, and that $\left\{\left(C, \mathscr{V}_{C}\right): C \in \mathscr{C}\right\}$ is a strongly compatible collection; we need to show that $\mathscr{U}^{\prime}$ is strongly complete. Since $\mathscr{U} \supseteq \mathscr{U}^{\prime} \supseteq \mathscr{U}^{2}, \mathscr{U}$ and $\mathscr{U}^{\prime}$ are weakly equivalent local uniformities. By Theorem $3.2, \mathscr{U}^{\prime}$ is strongly complete iff $\mathscr{U}$ is; hence it suffices to show that $\mathscr{U}$ is strongly complete. Choose $n \in \omega-\{0\}$ and let $\mathscr{G}$ be a weak Cauchy filter of degree $n$ on $(X, \mathscr{U})$. If some extension $\mathscr{F}$ of $\mathscr{G}$ converges to a point $x \in X$, then $x$ is an adherent point of $\mathscr{G}$, to which $\mathscr{G}$ converges by Theorem 3.2. So let $\mathscr{F}$ be an ultrafilter which extends $\mathscr{G}$; we shall show that $\mathscr{F}$ converges.

Let $U_{0}=\bigcup\{C \times C: C \in \mathscr{C}\} \in \mathscr{U}$. Choose $F \in \mathscr{F}$ so that $F \times F \subseteq U_{0}^{n}$; pick $x \in F$. Then $F \subseteq U_{0}^{n}[x]$. By the definition of $U_{0}$ and the star-finiteness of $\mathscr{C}, U_{0}^{n}[x]$ is the union of finitely many elements of $\mathscr{C}$. Since $\mathscr{F}$ is an ultrafilter, some given $C$ of $\mathscr{C}$ contained in $U_{0}^{n}[x]$ belongs to $\mathscr{F}$. Then it is easy to see that $\mathscr{F} \mid C$ must be weak Cauchy of degree $n$ in $U_{0}^{n}[C]$. Let $\mathscr{B}=\left\{B \in \mathscr{C}: B \subseteq U_{0}^{n}[C]\right\} ; \mathscr{B}$ is finite. Pick $V_{C} \in \mathscr{V}_{C}$. For each $n$-element sequence $\rho=\left\langle\rho_{0}, \ldots, \rho_{n-1}\right\rangle$ of elements of $\mathscr{B}$, we make choices as follows: By strong compatibility we have that $\mathscr{V}_{\rho_{n-1}}^{2 n}\left|C=\mathscr{V}_{C}^{2 n}\right| \rho_{n-1}$, so we may choose $V_{n-1, \rho} \in \mathscr{V}_{\rho_{n-1}}$ so that $V_{n-1, \rho}^{2 n} \cap C^{\times 2} \subseteq V_{C}^{2 n} \cap \rho_{n-1}^{\times 2} \subseteq V_{C}^{2 n}$. Similarly, we choose $V_{n-2, \rho} \in \mathscr{V}_{\rho_{n-2}}$ so that

$$
V_{n-2, \rho}^{2(n-1)} \cap \rho_{n-1}^{\times 2} \subseteq V_{n-1, \rho}^{2(n-1)} \cap \rho_{n-2}^{\times 2} \subseteq V_{n-1, \rho}^{2(n-1)},
$$

and so forth until we choose $V_{0, \rho} \in \mathscr{V}_{\rho_{0}}$ so that $V_{0, \rho}^{2} \cap \rho_{1}^{\times 2} \subseteq V_{1, \rho}^{2} \cap \rho_{0}^{\times 2} \subseteq V_{1, \rho}^{2}$. For each $B \in \mathscr{B}$ we now choose $W_{B} \in \mathscr{V}_{B}$ so that

$$
W_{B} \subseteq \bigcap\left\{V_{j, \rho}: j<n, \rho \in{ }^{n} \mathscr{B} \text {, and } B=\rho_{j}\right\} .
$$

For each $B \in \mathscr{C}$, let $\hat{W}_{B} \in \mathscr{V}_{B}$ be such that $\forall D \in \mathscr{B}$, $\hat{W}_{B} \cap D^{\times 2} \subseteq W_{D}$. Let $\hat{U}=$ $\bigcup\left\{\hat{W}_{B}: B \in \mathscr{C}\right\} \in \mathscr{U}$. Then

$$
\forall \rho \in{ }^{n} \mathscr{B}, \forall j<n, \quad \hat{U} \cap \rho_{j}^{\times 2}=\bigcup\left\{\hat{W}_{B} \cap \rho_{j}^{\times 2}: B \in \mathscr{C}\right\} \subseteq W_{\rho_{j}} \subseteq V_{j, \rho} .
$$

Now pick $F \in \mathscr{F} \mid \cup \mathscr{B}$ so that $F \subseteq C$ and $F \times F \subseteq \hat{U}^{n} \subseteq U_{0}^{n}$. Pick $x \in F$. For each $\rho \in{ }^{n \mathscr{B}}$, let

$$
S_{\rho}=\left\{y \in F: \exists x_{0}, \ldots, \exists x_{n} ; x_{0}=x, x_{n}=y \text {, and } \forall i<n,\left\langle x_{i}, x_{i+1}\right\rangle \in \hat{U} \cap \rho_{i}^{\times 2}\right\} .
$$

Then $F$ is represented as the finite union $F=\bigcup\left\{S_{\rho}: \rho \in{ }^{n} \mathscr{B}\right\}$. Since $\mathscr{F} \mid \bigcup \mathscr{B}$ is an ultrafilter, we may choose some $\rho \in{ }^{n} \mathscr{B}$ so that $S_{\rho} \in \mathscr{F} \mid \bigcup \mathscr{B}$. Pick $y, z \in S_{\rho}$. Then there are sequences $\left\langle y_{0}, \ldots, y_{n}\right\rangle$ and $\left\langle z_{0}, \ldots, z_{n}\right\rangle$ with $y_{0}=z_{0}=x, y_{n}=y$, and $z_{n}=z$ 
such that $\forall i\left\langle n,\left\langle y_{i}, y_{i+1}\right\rangle \in \rho_{i}\right.$, and $\left\langle z_{i}, z_{i+1}\right\rangle \in \rho_{i}$. We may now estimate the distance in $C$ from $y$ to $z$ as indicated:

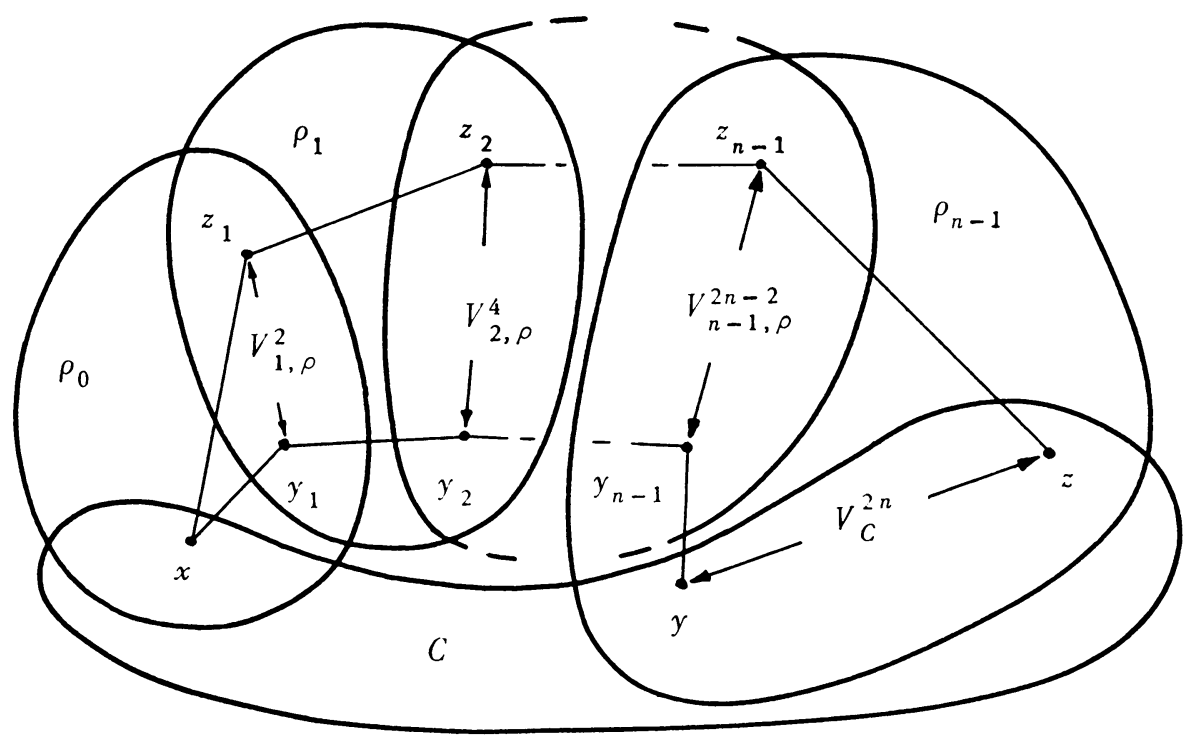

Hence $S_{\rho} \times S_{\rho} \subseteq V_{C}^{2 n}$. This shows $\mathscr{F} \mid C$ is a weak Cauchy filter of degree $2 n$ on $\left(C, \mathscr{V}_{C}\right)$, and hence converges.

In proving strong completeness in the above theorem, the assumption that $\left\{\left(C, \mathscr{V}_{C}\right): C \in \mathscr{C}\right\}$ is strongly compatible cannot be dropped, as shown by the following counterexample. Strong compatibility can, however, be replaced with the following weaker condition: $\left\{\left(C, \mathscr{V}_{C}\right): C \in \mathscr{C}\right\}$ is compatible and $\forall C, D \in \mathscr{C}$, $\forall n \in \omega-\{0\}, \exists m \in \omega-\{0\}: \mathscr{V}_{C}^{n}\left|D \supseteq \mathscr{V}_{D}^{m}\right| C$. The proof is similar to the one above, but messier. I do not know whether the star-finiteness condition is needed or not. It is perhaps worth mentioning that it may sometimes not be possible to choose $\mathscr{U}^{\prime}$ in the above theorem so that $\left(X, \mathscr{U}^{\prime}\right)$ is a strong extension of each $\left(C, \mathscr{V}_{C}\right)$, as is shown in the proof of Proposition 4.6.

4.5 Example. Of two compatible strongly complete locally uniform subspaces which cannot be pieced together to form a strongly complete locally uniform space.

Let $P=\omega-\{0\}$, let $X=P \times \omega$, let $A=P \times\{0,1\}$, and let $B=P \times P$, so that $X=A \cup B$. We shall now define an NLU $\mathscr{V}$ on $X$ whose relative topology is discrete, and whose restriction to $A$ or to $B$ is strongly complete. $\mathscr{V}$ will not be strongly complete although it is the strongest local uniformity whose restriction to $A$ or $B$ is $\mathscr{V} \mid A$ or $\mathscr{V} \mid B$ : Define $\phi: P \rightarrow P$ inductively by $\phi_{1}=1$ and $\phi_{j+1}=\phi_{j}+j$. Let $R$ be defined as follows:

$$
\begin{aligned}
R= & \left\{\left\langle\left\langle\phi_{1}, 0\right\rangle,\langle y, 1\rangle\right\rangle: \phi_{j} \leqq y<\phi_{j}+j, j \in P\right\} \\
& \cup\left\{\left\langle\left\langle\phi_{j}+j-1,1\right\rangle,\left\langle\phi_{j}+j-1, n\right\rangle\right\rangle: n \geqq 2, n, j \in P\right\} \\
& \cup\left\{\left\langle\left\langle\phi_{j}+j-1, n\right\rangle,\left\langle\phi_{n+j-1}+j-1,1\right\rangle\right\rangle: j>2, n, j \in P\right\} .
\end{aligned}
$$


For each $n \in P$, let

$$
V_{n}=\left[\left(R \cup R^{-1}\right) \cap(\{j \in \omega: j \geqq n\} \times \omega)^{\times 2}\right] \cup\{\langle x, x\rangle: x \in X\} .
$$

It is easy to see that $\left\{V_{n}: n \in \omega\right\}$ is a base for an NLU $\mathscr{V}$ on $X$ which generates the discrete topology. To see that $\mathscr{V} \mid A$ is complete notice first that $\left(\left(R \cup R^{-1}\right) \mid A\right)^{2}$ is an equivalence relation for which each equivalence class is finite. Similarly, one can show that $\mathscr{V} \mid B$ is complete by first verifying that $\left(\left(R \cup R^{-1}\right) \mid B\right)^{4}$ is an equivalence relation each of whose equivalence classes is made discrete by some $V_{n}$. On the other hand, $\mathscr{V}$ is not strongly complete since $\left\{\left\langle\phi_{j}, 0\right\rangle: j \in P\right\}$ is a weak Cauchy sequence of degree 4. Finally, $\mathscr{V}$ is the strongest common extension of $\mathscr{V} \mid A$ and $\mathscr{V} \mid B$ since $\forall n \in P, V_{n}=\left(V_{n} \cap A^{\times 2}\right) \cup\left(V_{n} \cap B^{\times 2}\right)$.

The result for uniformities analogous to Theorem 4.4 is false, as is shown by Proposition 4.6. It is curious, however, that in spite of this we still have the following result:

4.5 Proposition. If $X=X_{1} \cup X_{2}$, and $\left(X_{1}, \mathscr{U}_{1}\right)$ and $\left(X_{2}, \mathscr{U}_{2}\right)$ are compatible uniform spaces, then there is a uniformity $\mathscr{U}$ on $X$ such that $\mathscr{U} \mid X_{1}=\mathscr{U}_{1}$ and $\mathscr{U} \mid X_{2}=\mathscr{U}_{2}$.

Proof. $\forall U_{1} \in \mathscr{U}_{1}, \forall U_{2} \in \mathscr{U}_{2}$, let $U_{1} \square U_{2}=U_{1} \cup\left(U_{1} \circ U_{2}\right) \cup\left(U_{2} \circ U_{1}\right) \cup U_{2}$. Let $\mathscr{B}=\left\{U_{1} \square U_{2}: U_{1} \in \mathscr{U}_{1}, U_{2} \in \mathscr{U}_{2}\right\}$. Claim: $\mathscr{B}$ is a subbase for a uniformity of the required type. Pick $U_{1} \in \mathscr{U}_{1}$ and $U_{2} \in \mathscr{U}_{2}$; then it is clear that $\Delta(X) \subseteq U_{1} \square U_{2}$, and if $U_{1}$ and $U_{2}$ are symmetric, so is $U_{1} \square U_{2}$. In order to find $\hat{U}_{1} \in \mathscr{U}_{1}$ and $\hat{U}_{2} \in \mathscr{U}_{2}$ such that $\left(\hat{U}_{1} \square \hat{U}_{2}\right)^{2} \subseteq U_{1} \square U_{2}$, we make the following choices for $i, j=1,2 ; i \neq j$ : $P_{i} \in \mathscr{U}_{i}$ so that $P_{i}^{3} \subseteq U_{i} ; Q_{i} \in \mathscr{U}_{i}$ so that $Q_{i} \subseteq P_{i}$ and $Q_{i} \cap X_{j}^{\times 2} \subseteq P_{j} ; \hat{U}_{i} \in \mathscr{U}_{i}$ so that $\hat{U}_{i}^{2} \subseteq Q_{i}$. By definition, $\hat{U}_{1} \square \hat{U}_{2}$ is the union of four terms, and thus $\left(\hat{U}_{1} \square \hat{U}_{2}\right)^{2}$ will be the union of sixteen. It is a straightforward job to verify that each term is in fact a subset of $U_{1} \square U_{2}$. For example,

$$
\left(\hat{U}_{1} \circ \hat{U}_{2}\right) \circ\left(\hat{U}_{2} \circ \hat{U}_{1}\right) \subseteq \hat{U}_{1} \circ Q_{2} \circ \hat{U}_{1} \subseteq \hat{U}_{1} \circ P_{1} \circ \hat{U}_{1} \subseteq P_{1}^{3} \subseteq U_{1} \subseteq U_{1} \square U_{2} .
$$

Finally, for $i=1,2, \mathscr{B} \mid X_{i}$ is a base for $\mathscr{U}_{i}$ : Clearly, $\mathscr{B} \mid X_{i} \subseteq \mathscr{U}_{i}$, so it suffices to show that each $U_{i} \in \mathscr{U}_{i}$ has a subset in $\mathscr{B} \mid X_{i}$. Pick $U_{i} \in \mathscr{U}_{i}$. Choose $P_{i} \in \mathscr{U}_{i}$ so that $P_{i}^{2} \subseteq U_{i}$; choose $Q_{i} \in \mathscr{U}_{i}$ so that $Q_{i} \subseteq P_{i}$ and $Q_{i} \cap X_{j}^{\times 2} \subseteq P_{j}$. Then

$$
\begin{aligned}
\left(Q_{i} \square Q_{j}\right) \cap X_{i}^{\times 2} & =Q_{i} \cup\left(Q_{i} \circ\left(Q_{j} \cap X_{i}^{\times 2}\right)\right) \cup\left(\left(Q_{j} \cap X_{i}^{\times 2}\right) \circ Q_{i}\right) \cup\left(Q_{j} \cap X_{i}^{\times 2}\right) \\
& \subseteq P_{i}^{2} \subseteq U_{i} .
\end{aligned}
$$

Hence $\mathscr{B} \mid X_{i}$ is a base for $\mathscr{U}_{i}$.

4.6 Proposition. Suppose that $\mathscr{C}$ is a closed three-element cover of a space $X$, and that $\left\{\left(C, \mathscr{U}_{C}\right): C \in \mathscr{C}\right\}$ is a compatible collection of uniform spaces. There need not exist any uniformity $\mathscr{U}$ such that $\forall C \in \mathscr{C}, \mathscr{U} \mid C=\mathscr{U}_{C}$.

Proof by example. Let $\boldsymbol{C}$ be the complex numbers with the usual uniformity. Let $D$ be the unit disk minus the origin, assign it the relative uniformity of $C$. Let $A_{1}, B_{1}, C_{1}, A_{2}, B_{2}, C_{2}$ divide $D$ into six closed pieshaped pieces, as indicated. 


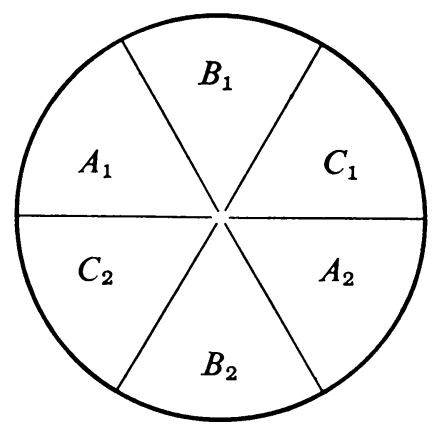

Assign each piece the relative uniformity of $D$. Let $\left(A, \mathscr{U}_{A}\right),\left(B, \mathscr{U}_{B}\right)$, and $\left(C, \mathscr{U}_{C}\right)$ be the independent sum of the uniformities for $A_{1}$ and $A_{2}, B_{1}$ and $B_{2}$, and $C_{1}$ and $C_{2}$, respectively. $\left\{\left(A, \mathscr{U}_{A}\right),\left(B, \mathscr{U}_{B}\right),\left(C, \mathscr{U}_{C}\right)\right\}$ is a compatible collection since, for instance, $\mathscr{U}_{A}\left|B=\mathscr{U}_{B}\right| A$ is the independent sum of the relative uniformities on $A_{1} \cap B_{1}$ and $A_{2} \cap B_{2}$. The collection is of course also strongly compatible since it consists of uniformities.

Finally, if $\mathscr{U}$ is a uniformity which restricts to $\mathscr{U}_{A}, \mathscr{U}_{B}$, and $\mathscr{U}_{C}$, then in particular, $\mathscr{U}$, viewed as a local uniformity, must be a strong extension of $\mathscr{U}_{A}$. Let $\mathscr{V}$ be any local uniformity piecing together $\mathscr{U}_{A}, \mathscr{U}_{B}$, and $\mathscr{U}_{C}$. Let $\mathscr{F}$ be the set of all deleted neighborhoods of the origin. It is easy to verify that $\mathscr{F}$ must be a weak Cauchy filter of degree $\leqq 4$ on $(D, \mathscr{V})$; but $\mathscr{F} \mid A$ is not a (weak) Cauchy filter on $\left(A, \mathscr{U}_{A}\right)$. Hence $\mathscr{V}$ is not a strong extension of $\mathscr{U}_{A}$ by Theorem 3.3. Hence no uniformity on $D$ restricts to $\mathscr{U}_{A}, \mathscr{U}_{B}$, and $\mathscr{U}_{C}$.

5. Bridge spaces. Here we shall be concerned with local uniformities whose topologies fail to be completely regular. Using results of the previous section, we shall first give a generalized construction and then specialize in order to verify the existence of particular kinds of locally uniform spaces, and to help show what conditions regular topological spaces may satisfy short of being completely regular.

To show that the constructed spaces are not completely regular we introduce a general class of topological spaces which we call bridge spaces, whose topology can never be completely regular, and verify that our constructions produce bridge spaces. Before going on, we shall show first that every regular space whose topology is not completely regular is already, in effect, half of a bridge space.

Definition. By a bridge space we shall mean a regular topological space $B$ together with a pair of points $a^{-}$and $a^{+}$in $B$ which have disjoint closures, but are functionally linked, that is, for any continuous real-valued function $f$ on $B, f\left(a^{-}\right)$ $=f\left(a^{+}\right)$.

5.1 Construction of a bridge space from a regular space which is not completely regular. Let $X$ be a regular, but not completely regular space. Choose $a \in X$ and an open neighborhood $N$ of $a$ so that there does not exist a continuous real-valued function on $X$ which is 0 at $a$ and 1 on $X-N$. Let $X^{-}$and $X^{+}$be two distinct 
copies of $X$. Let $B$ be the space obtained from $X^{-} \cup X^{+}$by identifying $x^{-}$and $x^{+}$, for each $x$ in the closed set $X-N . B$ is regular by Corollary 4.2.

Claim. $B$ with $a^{-}$and $a^{+}$is a bridge space. Suppose not, then there is a continuous function $f$ on $B$ such that $f\left(a^{-}\right)=0$ and $f\left(a^{+}\right)=1$. Define $\tau: B \rightarrow B$ by $\forall x^{-} \in X^{-} ; \tau\left(x^{-}\right)=x^{+}$; and $\forall x^{+} \in X^{+}, \tau\left(x^{+}\right)=x^{-}$. Let $g(x)=1-f(\tau(x))+f(x)$. It is clear that $\tau$ is continuous, that $g$ is continuous, that $g\left(a^{-}\right)=0$, and that $\forall x \in X-N$, $g\left(x^{-}\right)=g\left(x^{+}\right)=1$, but this is impossible by choice of $a$ and $N$. Therefore $B$ with $a^{-}$and $a^{+}$is a bridge space.

5.2 Construction $\left({ }^{4}\right)$ of a bridge space $S_{T}$ from a regular space $T$ which is not normal, using disjoint closed subsets $A$ and $B$ of $T$ which are not normally separated (that is, are not separable by disjoint open sets). Assume $T, A$, and $B$ are as above. Let $Z$ be the integers with the discrete topology; let $R_{T}$ be the space obtained from the product $T \times Z$ by identifying for each $n \in Z, x \in A$, and $y \in B,\langle x, 2 n\rangle$ with $\langle x, 2 n+1\rangle$ and $\langle y, 2 n-1\rangle$ with $\langle y, 2 n\rangle . R_{T}$ is regular by Corollary 4.2, since $\left\{[T \times\{n\}]^{\sim}: n \in Z\right\}$ is a locally finite closed cover of regular subspaces isomorphic with $T$, where $\sim$ denotes the quotient projection from $T \times Z$ onto $R_{T}$.

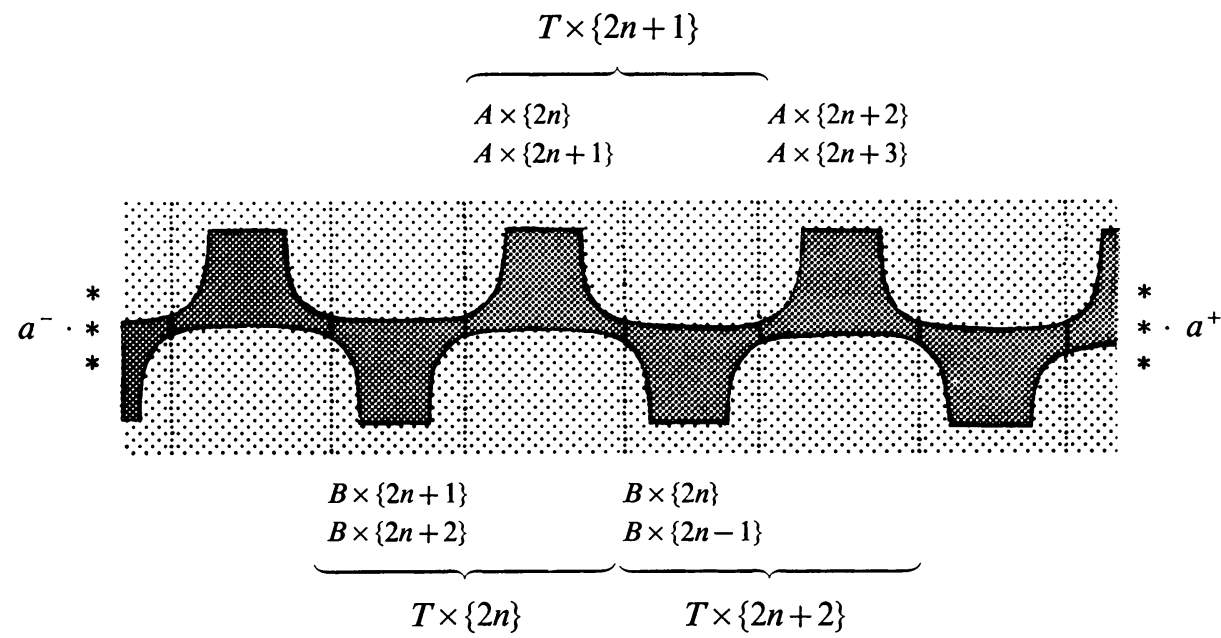

From now on, we shall use the points $\langle x, n\rangle$ of $T \times Z$ to represent the corresponding points $\langle x, n\rangle \sim$ of $R_{T}$, and dispense with $\sim$. Let $S_{T}$ be $R_{T}$ with two new points $a^{-}$and $a^{+}$added, and having, for each $k \in \omega$, the basic neighborhoods

$$
\begin{aligned}
& N_{k}^{-}=\left\{a^{-}\right\} \cup\{\langle x, y\rangle: x \in T, y<-k\}, \\
& N_{k}^{+}=\left\{a^{+}\right\} \cup\{\langle x, y\rangle: x \in T, y>k\} .
\end{aligned}
$$

$S_{T}$ is regular because first $R_{T}$ is and second $S_{T}$ is regular at $a^{-}$and $a^{+}$, since for each $k \in \omega$, the $S_{T}$-closure of $N_{k+1}^{ \pm}$is contained in $N_{k}^{ \pm}$.

$\left({ }^{4}\right)$ The method used here generalizes an example credited by Hewitt to Tychonoff and Arens; he describes the example in [7]. 
The fact that $S_{T}$ is really a bridge space will follow easily with the help of the next theorem. Like Berri and Sorgenfrey [1], we shall say that a filter is regular iff it has a base of open sets and a base of closed sets. The following two results generalize Propositions 4 and 5, p. 457 of [1].

5.3 Theorem. Let $T, A, B$, and $S_{T}$ be as in the above construction. Suppose $\mathscr{F}$ is a regular filter on $S_{T}$ such that for some $n \in Z$ and each $F \in \mathscr{F},(A \times\{n\}) \cap F^{-}$ and $B \times\{n\}$ are not normally separated in $T \times\{n\}$. Then $a^{-}$and $a^{+}$are adherent points of $\mathscr{F}$.

Proof. Let $T, A, B, S_{T}, \mathscr{F}$, and $n$ be as above. We shall show that $a^{+}$is an adherent point of $\mathscr{F}$, the proof for $a^{-}$being similar. Because of the identifications in $S_{T}$, it makes no difference to assume that $n$ is odd, for if $n$ were even, then $(A \times\{n\}) \cap F^{-}$ $=(A \times\{n+1\}) \cap F^{-}$, so that $(A \times\{n+1\}) \cap F^{-}$and $B \times\{n+1\}$ must be nonseparated in $T \times\{n+1\}$, for each $F \in \mathscr{F}$. It suffices to show by induction on odd integers that for each odd $m \geqq n$, and each $F \in \mathscr{F},(A \times\{m\}) \cap F^{-}$and $B \times\{m\}$ are not separated in $T \times\{m\}$. For each $j \in Z$, let $\iota_{j}: T \rightarrow T \times\{j\} \subseteq S_{T}$ be the obvious embedding; then for each open $P \subseteq S_{T}, \iota_{j}^{-1}[P]$ is open in $T$. For each $F \in \mathscr{F}$ we have that $A \cap \iota_{n}^{-1}\left[F^{-}\right]$and $B$ are not separated in $T$. By induction we assume for some odd integer $j \geqq n$ that $\forall F \in \mathscr{F}, A \cap \iota_{j}^{-1}\left[F^{-}\right]$and $B$ are not separated. Pick $F \in \mathscr{F}$, choose $G, H \in \mathscr{F}$ so that $H^{-} \subseteq G^{\circ} \subseteq G^{-} \subseteq F^{\circ}$. To show that $A \cap \iota_{j+2}^{-1}\left[F^{-}\right]$ and $B$ are not separated, we first establish that $A$ and $B \cap \iota_{j+1}^{-1}\left[G^{-}\right]$are not separated: Suppose to the contrary that $M$ and $N$ are disjoint open neighborhoods of $A$ and $B \cap \iota_{j+1}^{-1}\left[G^{-}\right]$. Then we have the following:

$$
\begin{gathered}
N \supseteq B \cap \iota_{j+1}^{-1}\left[G^{-}\right]=\iota_{j+1}^{-1}\left[G^{-} \cap(B \times\{j+1\})\right] \\
=\iota_{j}^{-1}\left[G^{-} \cap(B \times\{j\})\right]=\iota_{j}^{-1}\left[G^{-}\right] \cap B . \\
B=\left(B \cap \iota_{j}^{-1}\left[G^{-}\right]\right) \cup\left(B \cap \iota_{j}^{-1}\left[S_{T}-G^{-}\right]\right) \subseteq N \cup \iota_{j}^{-1}\left[S_{T}-G^{-}\right] . \\
A \cap \iota_{j}^{-1}\left[H^{-}\right] \subseteq M \cap \iota_{j}^{-1}\left[G^{\circ}\right] .
\end{gathered}
$$

So $M \cap \iota_{j}^{-1}\left[G^{\circ}\right]$ and $N \cup \iota_{j}^{-1}\left[S_{T}-G^{-}\right]$are disjoint open neighborhoods of $A \cap \iota_{j}^{-1}\left[H^{-}\right]$and $B$, contrary to assumption.

Finally, if $A \cap \iota_{j+2}^{-1}\left[F^{-}\right]$and $B$ were separated, we could repeat the above argument to obtain neighborhoods separating $A$ and $B \cap \iota_{j+1}^{-1}\left[G^{-}\right]$, which as we have just seen is impossible.

5.4 Corollary. Let $T$ be a regular space with disjoint closed subsets $A$ and $B$ which are not normally separated; then the space $S_{T}$ built from $T$ using $A$ and $B$ in Construction 5.2 is a bridge space.

Proof. Let $T, A, B$, and $S_{T}$ be as above. Suppose $f$ is a continuous real-valued function on $S_{T}$ such that $f\left(a^{+}\right)=1$. For each $r<1$, we may choose $n \in Z$ so that $\forall m \geqq n, \forall x \in T, f(x, m) \geqq r$, by continuity at $a^{+}$. For each $s<r$, let

$$
F_{s}=\{\langle x, j\rangle: f(x, j)>s\},
$$


then $\left\{F_{s}: s<r\right\}$ is a base for a regular filter $\mathscr{F}$ on $S_{T}$, by continuity of $f$. But for each $F \in \mathscr{F},(A \times\{n\}) \cap F^{-}=A \times\{n\}$, which is not separated from $B \times\{n\}$. Hence $a^{-}$is an adherent point of $\mathscr{F}$; so each neighborhood of $a^{-}$contains points whose image under $f$ is $\geqq s$, for each $s<r<1$. Consequently, $f\left(a^{-}\right) \geqq 1$. Similarly, $f\left(a^{-}\right) \leqq 1$.

5.5 Example of a bridge space $S_{T}$ which is countably compact, and is such that no $N L U$ generating the topology of $S_{T}$ has a completion. Assign the order topology to the ordinals $\Omega$ and $\Omega+1$. Let $T=\Omega \times(\Omega+1)$ be the product space. $T$ is countably compact since $\Omega$ is countably compact and $\Omega+1$ is compact. But $T$ is not normal, and the following closed subsets are not separated in $T ; A=\{\langle x, x\rangle: x \in \Omega\}$, and $B=\Omega \times\{\Omega\}$ (proofs of these properties are outlined in [9, pp. 131-132, 162-163]).

Let $S_{T}$ be the bridge space built from $T$ in Construction 5.2. $S_{T}$ is Hausdorff since $T$ is; therefore $S_{T}$ will be countably compact iff every infinite subset of $S_{T}$ has a limit point. Let $F$ be an infinite subset of $S_{T}$, then one of the following must be true:

(i) $\exists n \in Z$ such that $T \times\{n\}$ contains an infinite subset of $F$;

(ii) every neighborhood of $a^{-}$contains an infinite subset of $F$;

(iii) every neighborhood of $a^{+}$contains an infinite subset of $F$.

Hence $A$ must have a limit point in $T \times\{n\}$, for some $n$, or at $a^{-}$or $a^{+}$; so $S_{T}$ is countably compact.

Let $\mathscr{V}$ be an NLU which generates the topology of $S_{T} .\left(S_{T}, \mathscr{V}\right)$ cannot have a strong completion $\left(S_{T}^{*}, \mathscr{V}^{*}\right)$ since then $S_{T}$, being a countably compact subset of the strongly complete space $\left(S_{T}^{*}, \mathscr{V}^{*}\right)$, would be compact by Theorem 3.5 , which is impossible. By tracing down what is "wrong" with a specific Cauchy filter on $\left(S_{T}, \mathscr{V}\right)$, we can get the stronger result that $\mathscr{V}$ does not have a completion: We begin with a filter on $\Omega$,

$$
\mathscr{F}=\{F \subseteq \Omega: \exists x \in \Omega, \forall y>x ; y \in F\} .
$$

For each neighborhood $U$ of the diagonal in $\Omega \times \Omega$ we have (according to [9, p. 204]) $\exists x \in \Omega, \forall y, z>x:\langle x, y\rangle \in U$, so that $\mathscr{F}$ is a Cauchy filter on any NLU-space $(\Omega, \mathscr{U})$ which generates the order topology on $\Omega$. We transfer $\mathscr{F}$ to a filter $\mathscr{G}$ on $S_{T}$ by means of the map $\alpha \rightarrow\langle\alpha, \Omega, 0\rangle$. The image of $\Omega$ in $S_{T}$ is isomorphic with $\Omega$; hence $\mathscr{G}$ is a Cauchy filter for any NLU $\mathscr{V}$ which generates the topology of $S_{T}$. Now if $\left(S_{T}, \mathscr{V}\right)$ had a completion, $\mathscr{G}$ would converge in it to some point $y$. The neighborhood system of $y$, restricted to $S_{T}$, would be a regular filter $\mathscr{H}$ contained in $\mathscr{G}$. Each element of $\mathscr{H}$ must therefore contain a set of the form $\left.\{\langle\alpha, \Omega, 0\rangle: \alpha\rangle \alpha_{0}\right\}$, for some $\alpha_{0} \in \Omega$; this particular subset of $A \times\{0\}$ cannot be normally separated from $B \times\{0\}$. So by Theorem 5.3, $a^{-}$and $a^{+}$are adherent points of $\mathscr{H}$, but $\mathscr{H}$ cannot converge simultaneously to $a^{-}$and $a^{+}$, a contradiction. Hence $\left(S_{T}, \mathscr{V}\right)$ cannot have a completion.

5.6 Construction of a strongly complete $N L U$-space of cardinality $\boldsymbol{\aleph}_{1}$, whose relative topology is separable and first countable, but not completely regular. Our 
technique will be to construct an appropriate local uniformity on a bridge space obtained from Construction 5.2. Let $(T, \mathscr{U})$ be a complete uniform space of cardinality $\boldsymbol{\aleph}_{1}$ whose topology is separable and first countable but not normal (an example of such a space is given at the end of this construction).

Use $T$ in Construction 5.2 to get the bridge space $S_{T} . S_{T}$ is the countable union of the separable subspaces $\left\{a^{-}\right\},\left\{a^{+}\right\}, T \times\{n\}$, for $n \in Z$; hence $S_{T}$ is separable and of cardinality $\boldsymbol{\aleph}_{1}$. Also, $S_{T}$ is first countable since the neighborhood systems at $a^{-}$ and $a^{+}$have countable bases, and $R_{T}=S_{T}-\left\{a^{-}, a^{+}\right\}$has the following locally finite cover of first countable closed subspaces: $\{T \times\{k\}: k \in Z\}$. This collection is also star finite. For each $n \in \boldsymbol{Z}$ transfer the complete uniformity $\mathscr{U}$ to an isomorphic uniformity $\mathscr{U}_{n}$ on $T \times\{n\}$. The collection $\left\{\left(T \times\{n\}, \mathscr{U}_{n}\right): n \in Z\right\}$ is obviously compatible by construction, and since each $\mathscr{U}_{n}$ is a uniformity, the collection must be strongly compatible as well. So by Theorem 4.4 there is a strongest NLU $\mathscr{V}$ on $R_{T}$ such that $\forall n \in Z, \mathscr{V} \mid T \times\{n\}=\mathscr{U}_{n} . \mathscr{V}$ is also strongly complete.

Now define a semi-uniformity on $S_{T}$ as follows: $\forall V \in \mathscr{V}, \forall k \in \omega$, let $V_{k}=V$ $\cup\left(N_{k}^{-} \times N_{k}^{-}\right) \cup\left(N_{k}^{+} \times N_{k}^{+}\right)$. Let $\mathscr{W}$ be the semi-uniformity for which $\left\{V_{k}: k \in \omega\right.$, $V \in \mathscr{V}\}$ is a base. Claim: $\mathscr{W}$ is a strongly complete NLU generating the topology of $S_{T}$.

(1) $\mathscr{W}$ is a local uniformity generating the topology of $S_{T}$ : First, $\mathscr{W} \mid R_{T}$ is a local uniformity generating the topology of $R_{T}$ since for each $x \in R_{T}$, the sets $N_{k}^{-}$ and $N_{k}^{+}$may be taken small enough so that $V_{k}^{2}[x]=V^{2}[x]$, provided, for example, that $V$ is a subset of

$$
V^{*}=\bigcup\left\{(T \times\{n, n+1\})^{\times 2}: n \in Z\right\} .
$$

Also, it is clear that $\mathscr{W}$ generates the neighborhood systems at $a^{-}$and $a^{+} ; \mathscr{W}$ is locally uniform at $a^{-}$and $a^{+}$as well, since $\forall k \in \omega$,

$$
\left(V_{k-2}^{*} \circ V_{k-2}^{*}\right)\left[a^{ \pm}\right]=V_{k-2}^{*}\left[N_{k-2}^{ \pm}\right] \subseteq N_{k}^{ \pm} .
$$

(2) $\mathscr{W}$ is an NLU since each $V_{k}$ is a neighborhood of the diagonal in $S_{T} \times S_{T}$.

(3) $\mathscr{W}$ is strongly complete: Let $\mathscr{F}$ be a weak Cauchy filter on $\left(S_{T}, \mathscr{W}\right)$ of degree $n$. If $\exists k \in \omega, \forall F \in \mathscr{F}: F \cap\left(S_{T}-\left(N_{k}^{-} \cup N_{k}^{+}\right)\right) \neq \varnothing$, then $\mathscr{F}$ restricts to a filter on a subspace $X_{k}=T \times\{j \in Z:-k \leqq j \leqq k\}$, for some $k \in Z$. But then $\mathscr{F} \mid X_{k}$ is weak Cauchy of degree $n$ on the subspace $X_{k+2 n}=\left(V^{*}\right)^{n}\left[X_{k}\right]$. Now $\mathscr{W}$ was constructed so that $\left(X_{k+2 n}, \mathscr{W} \mid X_{k+2 n}\right)$ is a subspace of $\left(R_{T}, \mathscr{V}\right)$; thus $\mathscr{F} \mid X_{k}$ is weak Cauchy on $\left(R_{T}, \mathscr{V}\right)$ and hence converges. If $\forall k \in \omega, \exists F \in \mathscr{F}: F \cap\left(S_{T}-\left(N_{k}^{-} \cup N_{k}^{+}\right)\right)=\varnothing$, then $\mathscr{F}$ is eventually in every neighborhood of $a^{+}$or in every neighborhood of $a^{-}$, and therefore converges.

5.7 Example of a complete uniform space of cardinality $\aleph_{1}$ whose relative topology is separable and first countable but not normal. We shall get our example by constructing a complete uniformity for the half-open rectangle space. Let $\mathscr{U}$ be the usual uniformity for the unit interval $[0,1]$. For each $U \in \mathscr{U}$ and $x \in[0,1]$, let 
$U_{x}=U \cap\left([0, x]^{\times 2} \cup(x, 1]^{\times 2}\right)$. Let $\Gamma$ be the set of all monotone nonincreasing sequences on $[0,1]$. For each $\alpha \in \Gamma$, let $U_{\alpha}=\bigcap\left\{U_{x}: x \in \alpha\right\}$. The collection $\left\{U_{\alpha}: \alpha \in \Gamma, U \in \mathscr{U}\right\}$ is clearly a subbase for a semi-uniformity. To show that it is a subbase for a uniformity, pick $U \in \mathbb{Z}$ and $\alpha \in \Gamma$; then if we choose $V \in \mathbb{Z}$ so that $V^{2} \subseteq U$, we have the following: $\forall x, y \in \alpha ;\left(V_{x} \cap V_{y}\right)^{2}=V_{x}^{2} \cap V_{y}^{2} \subseteq U_{x} \cap U_{y}$. The same is obviously true for arbitrary intersections, and hence $V_{\alpha}^{2} \subseteq U_{\alpha}$.

Let $\mathscr{V}$ be the uniformity for which $\left\{U_{\alpha}: U \in \mathbb{I}, \alpha \in \Gamma\right\}$ is a subbase. We may show that the generated topology of $\mathscr{V}$ is the half-open interval topology for $[0,1]$ as follows: For each $x \in(0,1], \alpha \in \Gamma$, and $U \in \mathscr{U}$, we may choose $y \in(0,1] \cap U[x]$ so that $y<x$ and $\forall x_{n} \in \alpha, x_{n}<x$ implies $x_{n} \leqq y$; this assures that $(y, x] \subseteq U_{\alpha}[x]$. Hence the $\mathscr{V}$-topology is weaker than the half-open interval topology; and it is clearly stronger as well. The topology of $\mathscr{V}$, being the half-open interval topology, is thus separable and first countable. $\mathscr{V}$ is also complete: Let $\mathscr{F}$ be a $\mathscr{V}$-Cauchy filter on $[0,1]$. Then $\mathscr{F}$ is $\mathscr{O}$-Cauchy, and so converges in the usual topology to some point $x \in[0,1]$. Claim: $[0, x] \in \mathscr{F}$ and consequently $\mathscr{F}$ converges to $x$ in the half-open interval topology. Suppose not; let $\alpha$ be a strictly decreasing sequence which converges to $x$. Choose $F \in \mathscr{F}$ so that $F \times F \subseteq U_{\alpha}$, then for some $n \in \omega$, $F \subseteq\left(\alpha_{n+1}, \alpha_{n}\right]$, contradicting the fact that $\mathscr{F}$ converges to $x$.

Let $\mathscr{W}=\mathscr{V}(x) \mathscr{V}$ be the product uniformity on the unit square. $\mathscr{W}$ is complete by the product theorem for uniform spaces. The generated topology of $\mathscr{W}$, which is the half-open rectangle space topology, is separable and first countable since the half-open interval space is. However, the generated topology of $\mathscr{W}$ is not normal (see [9, pp. 59 and 133] for a summary of half-open rectangle space properties).

6. NLU-completeness. The examples and constructions of the previous section show that although strong completeness is a reasonable property for an NLU-space to have, one may not be able to construct a completion for some NLU-spaces. Consequently, we shall have to content ourselves with something less than the optimal completion result. It is still reasonable to ask, however, if one may easily describe the spaces for which completions or strong completions exist, and if one may always construct a "partial completion" of a space in which some well chosen class of filters converges. To discuss these questions we make the following

Definition. For any NLU-space $(X, \mathscr{V})$, an $N L U$-Cauchy filter on $(X, \mathscr{V})$ is a filter which converges in some larger NLU-space containing $(X, \mathscr{V}) .(X, \mathscr{V})$ is $N L U$-complete iff every NLU-Cauchy filter on $(X, \mathscr{V})$ converges in $(X, \mathscr{V})$. An $N L U$-completion of $(X, \mathscr{V})$ is an NLU-complete NLU-space containing $(X, \mathscr{V})$ as a dense subspace.

The above definition of an NLU-Cauchy filter is "external" in the sense that the local uniform structure of the space involved is not directly used; only its topological properties and its relation to other NLU-spaces are involved. So one thing we will need to find is an "internal" characterization of an NLU-Cauchy filter, given entirely in terms of the structure of the space involved. 
6.1 Theorem. Suppose that $(X, \mathscr{O})$ is a dense subspace of an $N L U$-space $(Y, \mathscr{V})$ and that every $N L U$-Cauchy filter on $(X, \mathscr{U})$ converges in $(Y, \mathscr{V})$. Then $(Y, \mathscr{V})$ is an $N L U$-completion of $(X, \mathscr{U})$.

Proof. Let $(X, \mathscr{U})$ and $(Y, \mathscr{V})$ be as supposed. Let $\mathscr{F}$ be an NLU-Cauchy filter on $(Y, \mathscr{V})$. Let $(Z, \mathscr{W})$ be an extension in which $\mathscr{F}$ converges to some point $z$. We may assume $Z=Y \cup\{z\}$. Let $\mathscr{N}$ be the restriction to $X$ of the neighborhood system of $z \mathscr{N}$ is a filter since $X$ is dense in $Z . \mathscr{N}$ is NLU-Cauchy since it converges to $z$; hence it converges to some point $y \in Y$. Let $M$ be a regular open neighborhood of $y$ in $Z$ (i.e. such that $M=M^{-\circ}$ ). Since $M \cap X \in \mathscr{N}$, there is an open neighborhood $N$ of $z$ such that $N \cap X=M \cap X$. We have $N^{-}=(N \cap X)^{-}=(M \cap X)^{-}$ $=M^{-}$, so that $z \in N \subseteq N^{-\circ}=M^{-\circ}=M$. Hence $M \cap Y \in \mathscr{F}$ since $\mathscr{F}$ converges to $z$. Since the neighborhood system at $y$ has a base of regular open sets, $\mathscr{F}$ converges to $y \in Y$. Thus $(Y, \mathscr{V})$ is an NLU-completion of $(X, \mathscr{U})$.

6.2 Lemma. Suppose $\mathscr{F}$ is an NLU-Cauchy filter on $(X, \mathscr{V})$; then $\mathscr{F}$ satisfies the following condition:

$$
\forall V \in \mathscr{V}, \exists U \in \mathscr{V}, \exists F \in \mathscr{F}:\left(U^{2}[F]\right)^{\times 2} \subseteq V .
$$

Proof. Let $(Y, \mathscr{W})$ be an NLU-space containing $(X, \mathscr{V})$ in which $\mathscr{F}$ converges to some point $y$. Pick $V \in \mathscr{V}$, let $\hat{V} \in \mathscr{W}$ be such that $\hat{V} \cap X^{\times 2} \subseteq V$. Choose $\hat{U} \in \mathscr{W}$ so that $\left(\hat{U}^{3}[y]\right)^{\times 2} \subseteq \hat{V}$, and choose $F \in \mathscr{F}$ so that $F \subseteq \hat{U}[y]$; let $U=\hat{U} \cap X^{\times 2}$. Then

$$
\left(U^{2}[F]\right)^{\times 2} \subseteq\left(\hat{U}^{2}[F]\right)^{\times 2} \cap X^{\times 2} \subseteq\left(\hat{U}^{3}[y]\right)^{\times 2} \cap X^{\times 2} \subseteq \hat{V} \cap X^{\times 2} \subseteq V .
$$

6.3 Lemma. Any NLU-space $\left(X, \mathscr{V}^{\circ}\right)$ may be embedded as a dense subspace of another $N L U$-space $\left(X^{*}, \mathscr{V}^{*}\right)$ in such a way that

(i) every filter on $(X, \mathscr{V})$ satisfying $\left({ }^{*}\right)$ converges in $\left(X^{*}, \mathscr{V}^{*}\right)$,

(ii) $\left(X^{*}, \mathscr{V}^{*}\right)$ is a strong extension of $(X, \mathscr{V})$.

Proof. In giving the proof it will be convenient to use small Greek letters for filters in place of the usual script letters. Let $X^{*}$ be the set of all those filters on $X$ which satisfy (*). We define a mapping $\sigma: X \rightarrow X^{*}$ by $x^{\sigma}=\{F: x \in F \subseteq X\}$, for each $x \in X$; in addition, if $A \subseteq X$ or $V \subseteq X \times X$, we set $A^{\sigma}=\left\{x^{\sigma}: x \in A\right\}$ and $V^{\sigma}=$ $\left\{\left\langle x^{\sigma}, y^{\sigma}\right\rangle:(x, y) \in V\right\}$. Define a map $\dagger: \mathscr{V} \rightarrow \mathscr{P}\left(X^{*} \times X^{*}\right)$ by

$$
V^{\dagger}=\left\{\langle\alpha, \beta\rangle: \alpha, \beta \in X^{*} \text { and } \exists A \in \alpha, \exists B \in \beta: A \times B \subseteq V\right\} .
$$

(1) First it is clear that $\sigma$ is one-to-one and that $\forall V \in \mathscr{V}, V^{\dagger} \cap\left(X^{\sigma} \times X^{\sigma}\right)=V^{\sigma}$.

(2) $\mathscr{V}^{\dagger}$ is a subbase for a semi-uniformity: First, each $V^{\dagger} \in \mathscr{V}^{\dagger}$ contains the diagonal in $\left(X^{*}\right)^{\times 2}$ since each filter $\alpha \in X^{*}$ satisfies $\left(^{*}\right)$ and so, in particular, $\forall V \in \mathscr{V}, \exists A \in \alpha: A \times A \subseteq V$. Second, each $V^{\dagger} \in \mathscr{V}^{\dagger}$ contains a symmetric element of $\mathscr{V}^{\dagger}$, since for each $V^{\dagger} \in \mathscr{V}^{\dagger}$ there is a symmetric $U \in \mathscr{V}$ contained in $V$, in which case $U^{\dagger} \subseteq V^{\dagger}$ and $U^{\dagger}$ is symmetric. 
(3) If $V, W \in \mathscr{V}$, then $V^{\dagger} \circ W^{\dagger} \subseteq(V \circ W)^{\dagger}$ : We have

$$
\begin{aligned}
V^{\dagger} \circ W^{\dagger} & =\{\langle\alpha, \gamma\rangle: \exists \beta(\exists A \in \alpha, \exists B \in \beta: A \times B \subseteq V) \\
& \left.\quad \text { and }\left(\exists B^{\prime} \in \beta, \exists C \in \gamma: B^{\prime} \times C \subseteq W\right)\right\} \\
& =\left\{\langle\alpha, \gamma\rangle: \exists \beta, \exists A \in \alpha, \exists B^{\prime \prime} \in \beta, \exists C \in \gamma: A \times B^{\prime \prime} \subseteq V \text { and } B^{\prime \prime} \times C \subseteq W\right\} \\
& \subseteq(V \circ W)^{\dagger} .
\end{aligned}
$$

(4) $\mathscr{V}^{\dagger}$ is a subbase for an NLU: We need to verify that $\forall \alpha \in X^{*}, \forall V \in \mathscr{V}$, $\exists U \in \mathscr{V}:\left(\left(U^{\dagger}\right)^{2}[\alpha]\right)^{\times 2} \subseteq V^{\dagger}$. By the previous step, it suffices to show that given $\alpha \in X^{*}$ and $V \in \mathscr{V}$ we may choose $U \in \mathscr{V}$ so that $\left(\left(U^{2}\right)^{\dagger}[\alpha]\right)^{\times 2} \subseteq V^{\dagger}$. We choose $D \in \alpha$ and a symmetric $U \in \mathscr{V}$ so that $\left(U^{2}[D]\right)^{\times 2} \subseteq V$. Now suppose $\beta, \gamma \in\left(U^{2}\right)^{\dagger}[\alpha]$; then there exist $A \in \alpha, B \in \beta$, and $C \in \gamma$ such that $A \subseteq D, A \times B \subseteq U^{2}$, and $A \times C \subseteq U^{2}$. Then $B \subseteq U^{2}[A]$ and $C \subseteq U^{2}[A]$, so that $B \times C \subseteq\left(U^{2}[A]\right)^{\times 2} \subseteq\left(U^{2}[D]\right)^{\times 2} \subseteq V$; consequently $(\beta, \gamma) \in V^{\dagger}$. This shows $\left(\left(U^{2}\right)^{\dagger}[\alpha]\right)^{\times 2} \subseteq V^{\dagger}$.

(5) From the previous steps, we may let $\mathscr{V}^{*}$ be the NLU for which $\mathscr{V}^{\dagger}$ is a subbase; it follows from (1) that $\sigma:(X, \mathscr{V}) \rightarrow\left(X^{*}, \mathscr{V}^{*}\right)$ is a uniform embedding. We next show that if $\alpha$ is a filter on $(X, \mathscr{V})$ which satisfies $\left({ }^{*}\right)$ then $\alpha^{\sigma}=\left\{A^{\sigma}: A \in \alpha\right\}$ converges to $\alpha \in\left(X^{*}, \mathscr{V}^{*}\right)$; this will verify property (i) of the lemma and also show that $\left(X^{\sigma}, \mathscr{V}^{\sigma}\right)$ is dense in $\left(X^{*}, \mathscr{V}^{*}\right)$. Given $V^{\dagger} \in \mathscr{V}^{\dagger}$, we pick $A \in \alpha$ so that $A \times A \subseteq V$; then $\forall x \in A,\left(x^{\sigma}, \alpha\right) \in V^{\dagger}$ since $\{x\} \times A \subseteq A \times A \subseteq V$; so $A^{\sigma} \subseteq V^{\dagger}[\alpha]$.

(6) Finally, $\left(X^{*}, \mathscr{V}^{*}\right)$ is a strong extension of $\left(X^{\sigma}, \mathscr{V}^{\sigma}\right)$ : Pick $n \in \omega-\{0\}$. Then $\left(\mathscr{V}^{\sigma}\right)^{n}$ has a base of elements of the form $\left(V^{\sigma}\right)^{n}$, and $\left(\mathscr{V}^{*}\right)^{n}$ has a subbase of elements of the form $\left(V^{\dagger}\right)^{n}$. By direct calculation we have $\left(V^{\dagger}\right)^{n} \cap\left(X^{\sigma}\right)^{\times 2} \supseteq\left(V^{\dagger} \cap\left(X^{\sigma}\right)^{\times 2}\right)^{n}$ $=\left(V^{\sigma}\right)^{n}$. Using induction we have first $V^{\dagger} \subseteq V^{\dagger}$; then $\forall j \in \omega-\{0\}$, if $\left(V^{\dagger}\right)^{j} \subseteq\left(V^{j}\right)^{\dagger}$, we have, using step (4),

$$
\left(V^{\dagger}\right)^{j+1}=V^{\dagger} \circ\left(V^{\dagger}\right)^{j} \subseteq V^{\dagger} \circ\left(V^{j}\right)^{\dagger} \subseteq\left(V^{j+1}\right)^{\dagger} .
$$

Hence

$$
\left(V^{\dagger}\right)^{n} \cap\left(X^{\sigma}\right)^{\times 2} \subseteq\left(V^{n}\right)^{\dagger} \cap\left(X^{\sigma}\right)^{\times 2}=\left(V^{n}\right)^{\sigma}=\left(V^{\sigma}\right)^{n} .
$$

Hence $\left(\mathscr{V}^{*}\right)^{n} \mid X=\left(\mathscr{V}^{\sigma}\right)^{n}=\left(\mathscr{V}^{*} \mid X\right)^{n}$, which is actually more than we needed to show.

6.4 TheOREM. A filter $\mathscr{F}$ on a space $(X, \mathscr{V})$ is $N L U$-Cauchy iff it satisfies $\left(^{*}\right)$.

Proof. This follows directly from the above two lemmas.

6.5 THEOREM. Every NLU-space has an NLU-completion which is also a strong extension.

Proof. Theorems 6.1 and 6.4 show that the extension given in Lemma 6.3 is the required NLU-completion.

A natural question is whether an NLU-space with a Hausdorff topology has a unique Hausdorff NLU-completion. In general the answer is no, and the following examples give two NLU-completions of the rational numbers, one of which is 
stronger than the uniform completion, the other weaker. Despite this, however, there is a uniqueness condition one can give for spaces which have a base of regular open sets.

6.6 Examples. Let $(Q, \mathscr{U})$ be the rational numbers with the usual uniformity. $\mathscr{U}$ has a base of sets $U_{n}=\left\{\langle x, y\rangle \in Q^{\times 2}:|x-y|<1 / n\right.$, for $\left.n \in \omega-\{0\}\right\}$. Let $\boldsymbol{R}$ be the real numbers; for each neighborhood $N$ of the diagonal in $\boldsymbol{R}^{\times 2}$, let $V_{N n}=U_{n}$ $\cup\left(N \cap\left(R^{\times 2}-Q^{\times 2}\right)\right)$. Let

$$
\mathscr{V}=\left\{V_{N n}: n \in \omega-\{0\} \text { and } N \text { is a neighborhood of the diagonal in } \boldsymbol{R}^{\times 2}\right\} .
$$

Let

$\mathscr{W}=\left\{V_{N n}: n \in \omega-\{0\}\right.$ and for some finite open $\operatorname{cover} \mathscr{C}$ of $\left.R, N=\bigcup\left\{C^{\times 2}: C \in \mathscr{C}\right\}\right\}$.

Then $\mathscr{V}$ and $\mathscr{W}^{r}$ are bases for NLU's which give the usual topology on $\boldsymbol{R}$, and so every Cauchy filter on $(Q, \mathscr{U})$ converges in $(\boldsymbol{R}, \mathscr{V})$ and in $(\boldsymbol{R}, \mathscr{W})$. By Theorem 6.1, $(\boldsymbol{R}, \mathscr{V})$ and $(\boldsymbol{R}, \mathscr{W})$ are bases for NLU-completions of $(Q, \mathscr{U})$. But the local uniformity for $\mathscr{V}$ is strictly stronger than the usual uniformity, and that of $\mathscr{W}$ strictly weaker.

6.7 Lemma. Suppose $X$ is a dense subspace of the topological space $\hat{X}$ and $A$ is a regular open set in $X$, then $A^{-\wedge \circ \wedge} \cap X=A$, where ${ }^{-\wedge}$ and ${ }^{\circ \wedge}$ denote the closure and interior operations on $\hat{X}$.

Proof. Let $A, X, \hat{X},{ }^{-\wedge},{ }^{\circ \wedge}$ be as above, let ${ }^{-}$and ${ }^{\circ}$ denote the closure and interior operations in $X . \hat{X}-A^{-\wedge}$ is open, hence

So

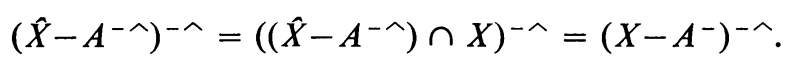

and

$$
A^{-\wedge \circ \wedge}=\hat{X}-\left(X-A^{-}\right)^{-\wedge}
$$

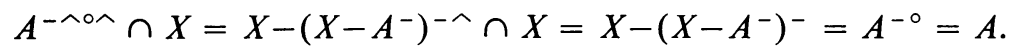

6.8 TheOREM. Suppose $(X, \mathscr{V})$ is an NLU-space whose topology is Hausdorff. Then any two Hausdorff completions of $(X, \mathscr{V})$ have homeomorphic topologies. If, in addition, $(X, \mathscr{V})$ has a base of regular open sets, then it has a unique Hausdorff $N L U$-completion with the same property.

Proof. Let $(\hat{X}, \hat{\mathscr{V}})$ and $\left(X^{*}, \mathscr{V}^{*}\right)$ be two Hausdorff NLU-completions of an NLU-space $(X, \mathscr{V})$ which has a Hausdorff topology. Let ${ }^{-}$and ${ }^{\circ},{ }^{-\wedge}$ and ${ }^{\circ \wedge}$, and -* and ${ }^{\circ *}$ denote the closure and interior operators for the spaces $(X, \mathscr{V}),(\hat{X}, \hat{\mathscr{V}})$, and $\left(X^{*}, \mathscr{V}^{*}\right)$ respectively. Let $\iota: X \rightarrow X$ be the identity map; let $\imath$ be the closure of $\iota \subseteq X \times X$ in $\hat{X} \times X^{*}$. Then $\forall x \in \hat{X}-X$, there is a filter $\mathscr{F}$ on $X$ converging to $x$; but $\mathscr{F}$ also converges to a unique point $y \in X^{*}-X$. Hence the filter base $\{A \times B: A, B \in \mathscr{F}\}$ converges to the unique point $\langle x, y\rangle \in \hat{X} \times X^{*}$. This shows that $i$ is a function which is $1-1$. Similarly, $i^{-1}$ is a function. By the above lemma, 
the complement of a regular open set in $\hat{X}$ has the form $\hat{X}-A^{-\wedge \circ \wedge}=\left(\hat{X}-A^{-\wedge}\right)^{-\wedge}$ $=\left(X-A^{-}\right)^{-\wedge}$, with $A \subseteq X$. But $i\left[\left(X-A^{-}\right)^{-\wedge}\right]=\left(X-A^{-}\right)^{-*}$ is then the complement of a regular open set in $X^{*}$. We conclude that $i$ preserves regular open sets, and so is open by regularity of $\hat{X}$. Similarly, $i^{-1}$ is open. Hence $i$ is a homeomorphism.

Now suppose $\mathscr{V}$ has a base of regular open sets. We will first show that there is at most one Hausdorff NLU-completion with a base of regular open sets: Suppose $\left(X^{*}, \mathscr{V}^{*}\right)$ is a Hausdorff NLU-completion with a base $\mathscr{B}$ of regular open sets. If $W \in \mathscr{B}$ and $V=W \cap X^{\times 2}$, then since $X^{\times 2}$ is dense in $\left(X^{*}\right)^{\times 2}$, we have $V^{-* \circ *}$ $=W^{* * *}=W . W$ is thus determined by its restriction to $X$ and the topology of $X^{*}$, which is unique as we have just seen. Hence $\mathscr{V}^{*}$ is unique.

To show the existence of such a completion, let $(\hat{X}, \hat{\mathscr{V}})$ be any NLU-completion of $(X, \mathscr{V})$. Let $\mathscr{B}$ be the set of regular open neighborhoods $U$ of the diagonal in $\hat{X}^{\times 2}$ such that $U \cap X^{\times 2} \in \mathscr{V}$. Let $\mathscr{V}^{*}$ be the semi-uniformity for which $\mathscr{B}$ is a base. By the above lemma, each regular open element of $\mathscr{V}$ is the restriction of an element of $\mathscr{R}$. Hence $\mathscr{V}^{*} \mid X=\mathscr{V}$. For each $V \in \hat{\mathscr{V}}, V^{3} \supseteq V^{-\wedge} \supseteq V^{-\wedge \circ \wedge} \in \mathscr{V}^{*}$; thus $\mathscr{V}^{*}$ is stronger than $\hat{\mathscr{V}}^{3}$ and so generates the topology of $\hat{X}$. By Theorem 1.9, $\mathscr{V}^{*}$ is an NLU. Every NLU-Cauchy filter on $(X, \mathscr{V})$ converges in $\left(\hat{X}, \mathscr{V}^{*}\right)$. Hence $\left(\hat{X}, \mathscr{V}^{*}\right)$ is an NLU-completion of $(X, \mathscr{V})$ by Theorem 6.1 .

We now return to the question of when an NLU-space has a completion or a strong completion. Our discussion will involve the following two conditions on a space $(X, \mathscr{V})$ :

(i) Every weak Cauchy filter on $(X, \mathscr{V})$ is Cauchy;

(ii) every Cauchy filter on $(X, \mathscr{V})$ is an NLU-Cauchy filter.

Notice that if a space $(X, \mathscr{V})$ satisfies (i), then for each Cauchy filter $\mathscr{F}$ on $X$ the filter $\mathscr{F}^{\prime}=\left\{V^{2}[F]: F \in \mathscr{F}, V \in \mathscr{V}\right\}$ is weak Cauchy, and therefore Cauchy; hence $\mathscr{F}$ satisfies the condition $\left({ }^{*}\right)$ of Theorem 6.3 , and is thus NLU-Cauchy. Therefore $(X, \mathscr{V})$ satisfies (ii). Condition (ii) is strictly weaker than (i) and in fact the space $(Y, \mathscr{V})$ of Example 3.4 satisfies (ii) but not (i).

6.10 Theorem. An NLU-space $(X, \mathscr{V})$ has a strong completion iff every weak Cauchy filter on $(X, \mathscr{V})$ is Cauchy; in this case, if $(\hat{X}, \hat{\mathscr{V}})$ is an NLU-completion of $(X, \mathscr{V})$ and is also a strong extension, then $(\hat{X}, \hat{\mathscr{V}})$ is a strong completion.

Proof. If $(X, \mathscr{V})$ has a strong completion, then by definition, each weak Cauchy filter is NLU-Cauchy and hence Cauchy. Now suppose each weak Cauchy filter is Cauchy. By Theorem 6.5 there is an NLU-completion $(\hat{X}, \hat{\mathscr{V}})$ of $(X, \mathscr{V})$ which is also a strong extension. Let $\mathscr{F}$ be a weak Cauchy filter of degree $k$ on $(\hat{X}, \hat{\mathscr{V}})$; let $\mathscr{G}=\{\hat{V}[F] \cap X: \hat{V} \in \hat{\mathscr{V}}, F \in \mathscr{F}\}$. Then $\mathscr{G}$ is a filter on $X$ which is weak Cauchy of degree $k+2$ in $(\hat{X}, \hat{\mathscr{V}})$. Since $(\hat{X}, \hat{\mathscr{V}})$ is a strong extension, $\mathscr{G}$ is weak Cauchy on $(X, \mathscr{V})$ as well and thus converges in $X$ to some point $y$. Then $y$ is an adherent point of $\mathscr{F}$ to which $\mathscr{F}$, being weak Cauchy, must converge, by Theorem 3.3. Thus $(\hat{X}, \hat{\mathscr{V}})$ is a strong completion of $(X, \mathscr{V})$. 
I do not know whether every NLU-space satisfying (ii) has a completion; the following example, however, does answer a similar question.

6.11 Example of an NLU-space with a Hausdorff topology on which every Cauchy filter is NLU-Cauchy, but whose strongest Hausdorff NLU-completion is not complete. Let $X=\Omega \times \Omega$; let $\hat{X}=\Omega \times(\Omega \cup\{\Omega\})$. We assign $\hat{X}$ a topology as follows: Let $X$ have the discrete topology, and for each $\alpha \in \Omega$ let the basic neighborhoods of $\langle\alpha, \Omega\rangle$ be of the form $[\mu, \alpha] \times(\lambda, \Omega]$, with $\lambda, \mu \in \Omega$, and $\mu<\alpha$ or $\mu=0$. This topology for $\hat{X}$ is completely regular since the neighborhood system at each point has a base of clopen sets. Hence the collection $\hat{\mathscr{V}}$ of all neighborhoods of the diagonal in $\hat{X}^{\times 2}$ is an NLU.

Let $\mathscr{V}=\hat{\mathscr{V}} \mid X$; let $\Delta(X)$ be the diagonal in $X^{\times 2}$. We shall now determine the Cauchy filters on $(X, \mathscr{V})$ : Suppose $\mathscr{F}$ is a Cauchy filter on $(X, \mathscr{V})$ such that $\bigcap \mathscr{F}=\varnothing$. First, it is easy to see that $\forall F \in \mathscr{F}$, sup $\left\{x_{2}: \dot{x} \in F\right\}=\Omega$. Next suppose $\alpha_{0}$ is the least ordinal such that for some $F \in \mathscr{F}$, $\sup \left\{x_{1}: x \in F\right\}=\alpha_{0}$. If $\alpha_{0}<\Omega$, then $\left\langle\alpha_{0}, \Omega\right\rangle \in \hat{X}$ must be an adherent point of $\mathscr{F}$. Hence $\mathscr{F}$, being Cauchy, converges to $\left\langle\alpha_{0}, \Omega\right\rangle$. If $\alpha_{0}=\Omega$, that is if $\forall F \in \mathscr{F}, \forall \alpha \in \Omega, \exists x \in F: x_{1}>\alpha$, we may derive a contradiction as follows: Let

$$
V_{0}=\Delta(X) \cup \bigcup\left\{([0, \alpha] \times[\alpha, \Omega))^{\times 2}: \alpha \in \Omega\right\} .
$$

Then $V_{0} \in \mathscr{V}$ and we may choose $F \in \mathscr{F}$ so that $F \times F \subseteq V_{0}$; pick $x \in F$, choose $y \in F$ so that $y_{1}>x_{2}$; then since $\langle x, y\rangle \in V_{0}$, there exists $\alpha \in \Omega$ such that $x_{1}, y_{1} \leqq \alpha$ and $x_{2}, y_{2} \geqq \alpha$. Thus $x_{2} \geqq \alpha \geqq y_{1}>x_{2}$, a contradiction.

We have thus shown that every nonconvergent Cauchy filter on $X$ converges to a point in $\hat{X}$, and is therefore NLU-Cauchy. Since $X$ is dense in $\hat{X},(\hat{X}, \hat{\mathscr{V}})$ is an NLU-completion of $(X, \mathscr{V})$ by Theorem 6.1. By construction, $\hat{\mathscr{V}}$ generates the order topology on $\hat{X}-X=\Omega \times\{\Omega\}$, and $\hat{\mathscr{V}} \mid(\hat{X}-X)$ consists of all neighborhoods of the diagonal in $(\hat{X}-X)^{\times 2}$. Consequently $\hat{\mathscr{V}} \mid(\hat{X}-X)$ is the unique uniformity which generates this order topology; $(\hat{X}-X, \hat{\mathscr{V}} \mid(\hat{X}-X))$ thus has a nonconvergent Cauchy filter $\hat{\mathscr{F}}$ which would converge to the omitted point $\langle\Omega, \Omega\rangle$.

Finally, $(\hat{X}, \hat{\mathscr{V}})$ is the strongest Hausdorff NLU-completion of $(X, \mathscr{V})$ : Since $(\hat{X}, \hat{\mathscr{V}})$ has a Hausdorff topology, its topology must be the same as that of the strongest Hausdorff NLU-completion by Theorem 6.8. But since $\hat{\mathscr{V}}$ consists of all neighborhoods of the diagonal in $\hat{X}^{\times 2}$, it must be the strongest completion itself.

We say that a uniformly continuous function $f:(X, \mathscr{U}) \rightarrow(Y, \mathscr{V})$ preserves NLU-Cauchy filters iff the image under $f$ of each NLU-Cauchy filter on $(X, \mathscr{U})$ extends to an NLU-Cauchy filter on $(Y, \mathscr{V})$. Notice that the nonconvergent Cauchy filter $\hat{\mathscr{F}}$ in the above example is an NLU-Cauchy filter in the closed subspace $(\hat{X}-X, \hat{\mathscr{V}} \mid(\hat{X}-X))$ since $\hat{\mathscr{V}} \mid(\hat{X}-X)$ is a uniformity. We have just proved the following unfortunate facts:

6.12 CoRollary. An inclusion map from a subspace of an NLU-space need not preserve NLU-Cauchy filters; a closed subspace of an NLU-complete space need not be NLU-complete. 
In what cases, then, can one conclude that a closed subspace of an NLUcomplete space is NLU-complete? One somewhat restrictive possibility is the following:

Definition. Suppose $(X, \mathscr{U})$ is a subspace of $(Y, \mathscr{V})$. Then $(Y, \mathscr{V})$ is a conditions preserving extension of $(X, \mathscr{U})$ iff there is a subbase $\mathscr{B}$ for $\mathscr{V}$ such that $\forall x \in X$, $\forall \hat{U} \in \mathscr{B}, \forall V \in \mathscr{U}$, if $\left(V^{2}[x]\right)^{\times 2} \subseteq \hat{U}$, then $\exists \hat{V} \in \mathscr{B}:\left(\hat{V}^{2}[x]\right)^{\times 2} \subseteq \hat{U}$ and $\hat{V} \cap X^{\times 2}=V$.

6.13 Lemma. Suppose $(Y, \mathscr{V})$ is a conditions preserving extension of $(X, \mathscr{U})$ and $\mathscr{F}$ is an NLU-Cauchy filter on $(X, \mathscr{U})$; then $\mathscr{F}$ is NLU-Cauchy in $(Y, \mathscr{V})$.

Proof. Let $\mathscr{B}$ be a subbase for $\mathscr{V}$ such that $\forall x \in X, \forall \hat{U} \in \mathscr{B}, \forall V \in \mathscr{V}$, if $\left(V^{2}[x]\right)^{\times 2}$ $\subseteq U$, then $\exists \hat{V} \in \mathscr{B}:\left(\hat{V}^{2}[x]\right)^{\times 2} \subseteq \hat{U}$ and $\hat{V} \cap X^{\times 2}=V$. Let $\mathscr{F}$ be an NLU-Cauchy filter on $(X, \mathscr{U})$. Pick $\hat{U} \in \mathscr{B}$. By Theorem 6.4 we may choose $V \in \mathscr{U}$ and $F \in \mathscr{F}$ so that $\left(V^{2}[F]\right)^{\times 2} \subseteq \hat{U}$, and we may choose $W \in \mathscr{U}$ and $G \in \mathscr{F}$ so that $\left(W^{2}[G]\right)^{\times 2} \subseteq V$ and $W \subseteq V$ and $G \times G \subseteq W$. Pick $y \in G \cap F$. We may choose $P \in \mathscr{B}$ so that $P \cap X^{\times 2}$ $=V$ and $\left(P^{2}[y]\right)^{\times 2} \subseteq \hat{U}$. Then $\left(W^{2}[y]\right)^{\times 2} \subseteq P$, and we may choose $Q \in \mathscr{B}$ so that $Q \cap X^{\times 2}=W$ and $\left(Q^{2}[y]\right)^{\times 2} \subseteq P$. Let $\hat{W}=P \cap Q ;$ then $\hat{W} \cap X^{\times 2}=V \cap W=W$. Since $G \times G \subseteq W$, we have $G \subseteq W[y] \subseteq \hat{W}[y]$ and

$$
\left(\hat{W}^{2}[G]\right)^{\times 2} \subseteq\left(\hat{W}^{3}[y]\right)^{\times 2} \subseteq\left(P\left[Q^{2}[y]\right]\right)^{\times 2} \subseteq\left(P^{2}[y]\right)^{\times 2} \subseteq \hat{U} .
$$

Hence $\mathscr{F}$ is NLU-Cauchy on $(Y, \mathscr{V})$, by Theorem 6.4 .

DEFINITION. We shall say that a subspace $X$ of a topological space $Y$ is essentially closed iff for each $y$ in the closure of $X$, the $Y$-neighborhood system of $y$ restricted to $X$ converges in $X$.

6.14 Theorem. Let $(X, \mathscr{U})$ be a subspace of an $N L U$-space $(Y, \mathscr{V})$. If $(X, \mathscr{U})$ is $N L U$-complete, then it is essentially closed. Suppose on the other hand that $(X, \mathscr{U})$ is essentially closed in $(Y, \mathscr{V})$. If $(Y, \mathscr{V})$ is (strongly) complete then so is $(X, \mathscr{U})$; if $(Y, \mathscr{V})$ is an NLU-complete conditions preserving extension of $(X, \mathscr{U})$, then $(X, \mathscr{U})$ is NLU-complete.

Proof. Let $(X, \mathscr{U})$ be a subspace of $(Y, \mathscr{V})$. If $(X, \mathscr{U})$ is NLU-complete, and if $y$ belongs to the $Y$-closure of $X$, then $y$ generates an NLU-Cauchy filter on $X$ which converges in $X$. Now suppose $(X, \mathscr{U})$ is essentially closed in $(Y, \mathscr{V})$. If $(Y, \mathscr{V})$ is (strongly) complete and $\mathscr{F}$ is a (weak) Cauchy filter on $(X, \mathscr{U})$, then $\mathscr{F}$ is (weak) Cauchy in $(Y, \mathscr{V})$, and hence converges in the closure of $X$. Thus it also converges in $X$. Hence $(X, \mathscr{U})$ is (strongly) complete. If $(Y, \mathscr{V})$ is an NLU-complete conditions preserving extension of $(X, \mathscr{U})$, then an NLU-Cauchy filter on $(X, \mathscr{U})$ will be NLU-Cauchy in $(Y, \mathscr{V})$ and hence converge in the closure of $X$, and therefore in $X$ itself; thus $(X, \mathscr{U})$ is NLU-complete.

Finally, as an application of our ideas about completeness, we shall consider products of NLU-spaces. But first we shall sketch a few facts about products. As with uniform spaces, one can easily verify that for any indexed collection 
$\left\{\left(X_{\alpha}, \mathscr{V}_{\alpha}\right): \alpha \in \Gamma\right\}$ of NLU-spaces, there is a unique product space in the category of NLU-spaces and uniform functions whose underlying set is $X\left\{X_{\alpha}: \alpha \in \Gamma\right\}$. Its structure has a subbase of sets

$$
W_{\alpha, V}=\left\{\langle f, g\rangle \in\left(\times\left\{X_{\alpha}: \alpha \in \Gamma\right\}\right)^{\times 2}:\langle f(\alpha), g(\alpha)\rangle \in V\right\},
$$

for $\alpha \in \Gamma$ and $V \in \mathscr{V}_{\alpha}$. Just as with uniform spaces, the product of the generated topologies of the $\left(X_{\alpha}, \mathscr{V}_{\alpha}\right)$ is the generated topology of the product

$$
\times\left\{\left(X_{\alpha}, \mathscr{V}_{\alpha}\right): \alpha \in \Gamma\right\} .
$$

For each $\alpha \in \Gamma$ and each $f \in X\left\{X_{\alpha}: \alpha \in \Gamma\right\}$, we define a coordinate embedding

$$
\iota_{f, \alpha}:\left(X_{\alpha}, \mathscr{V}_{\alpha}\right) \rightarrow \times\left\{\left(X_{\alpha}, \mathscr{V}_{\alpha}\right): \alpha \in \Gamma\right\}
$$

by requiring that $\forall \beta \in \Gamma, \iota_{f, \alpha}(x)(\beta)$ equals $x$ if $\beta=\alpha$, and equals $f(\alpha)$ otherwise. Each $\iota_{f, \alpha}$ is clearly one-to-one, and it is an isomorphic embedding as well since for each $\langle x, y\rangle \in X_{\alpha}^{\times 2}$ and each $V \in \mathscr{V}_{\alpha},\langle x, y\rangle \in V$ iff $\left\langle\iota_{f, \alpha}(x), \iota_{f, \alpha}(y)\right\rangle \in W_{\alpha, V}$; this is true by definition of $W_{\alpha, V}$ and $\iota_{f, \alpha}$.

The product $\times\left\{\left(X_{\alpha}, \mathscr{V}_{\alpha}\right): \alpha \in \Gamma\right\}$ is a strong extension of each $\iota_{f, \alpha}\left[X_{\alpha}\right]$ since $\forall n \in \omega-\{0\}, W_{\alpha, V}^{n}=W_{\alpha, V^{n}}$, so that

$$
\tilde{\imath}_{f, \alpha}\left[V^{n}\right]=W_{\alpha, V^{n}} \cap\left(\iota_{f, \alpha}\left[X_{\alpha}\right]\right)^{\times 2}=W_{\alpha, V}^{n} \cap\left(\iota_{f, \alpha}\left[X_{\alpha}\right]\right)^{\times 2} .
$$

Similarly, $X\left\{\left(X_{\alpha}, \mathscr{V}_{\alpha}\right): \alpha \in \Gamma\right\}$ is a conditions preserving extension of each $\iota_{f, \alpha}\left[X_{\alpha}\right]$ since if $x \in X_{\alpha}, V \in \mathscr{V}_{\alpha}$, and $U \in \mathscr{V}_{\alpha}$, and

$$
\left(\left(\tilde{\imath}_{f, \alpha}[V]\right)^{2}\left[\iota_{f, \alpha}(x)\right]\right)^{\times 2}=\left(\iota_{f, \alpha}\left[V^{2}[x]\right]\right)^{\times 2} \subseteq W_{\alpha, U},
$$

then $\left(V^{2}[x]\right)^{\times 2} \subseteq U$, so that

$$
\left(W_{\alpha, V}^{2}\left[\iota_{f, \alpha}(x)\right]\right)^{\times 2} \subseteq W_{\alpha, U} \quad \text { and } \quad W_{\alpha, V} \cap\left(\iota_{f, \alpha}[X]\right)^{\times 2}=\tilde{\imath}_{f, \alpha}[V] .
$$

Finally, $\iota_{f, \alpha}\left[X_{\alpha}\right]$ is essentially closed in $X\left\{X_{\alpha}: \alpha \in \Gamma\right\}:$ Suppose $\mathscr{F}$ is a filter on $\iota_{f, \alpha}\left[X_{\alpha}\right]$ which converges in $\left(\iota_{f, \alpha}\left[X_{\alpha}\right]\right)^{-}$to some point $g$. Then for each $\beta \neq \alpha$, the projection $p_{\beta}[\mathscr{F}]$ converges in $\{f(\beta)\}^{-}$to $g(\beta)$, and hence to $f(\beta) ; p_{\alpha}[\mathscr{F}]$ converges to $g(\alpha)$. Hence $\mathscr{F}$ converges to $\iota_{f, \alpha}(g(\alpha))$.

6.15 TheOREM. Let $\left\{\left(X_{\alpha}, \mathscr{V}_{\alpha}\right): \alpha \in \Gamma\right\}$ be an indexed collection of NLU-spaces; then $\times\left\{\left(X_{\alpha}, \mathscr{V}_{\alpha}\right): \alpha \in \Gamma\right\}$ is $N L U$-complete, complete, or strongly complete iff each $\left(X_{\alpha}, \mathscr{V}_{\alpha}\right)$ is.

Proof. Let $\left\{\left(X_{\alpha}, \mathscr{V}_{\alpha}\right): \alpha \in \Gamma\right\}$ be an indexed collection of NLU-spaces; let $\left\{W_{\alpha, V}: \alpha \in \Gamma, V \in \mathscr{V}_{\alpha}\right\}$ be the subbase for the product given above; let $f$ be an arbitrary element of $\times\left\{X_{\alpha}: \alpha \in \Gamma\right\}$. For each $\alpha \in \Gamma$ let $\iota_{f, \alpha}$ be the coordinate embedding defined above, and let $p_{\alpha}$ be the projection map onto $X_{\alpha}$.

(1A) Suppose each $\left(X_{\alpha}, \mathscr{V}_{\alpha}\right)$ is NLU-complete. Let $\mathscr{F}$ be an NLU-Cauchy filter 
on $X\left\{\left(X_{\alpha}, \mathscr{V}_{\alpha}\right): \alpha \in \Gamma\right\}$. Then for each $\alpha$, and each $U \in \mathscr{V}_{\alpha}$, there is a $V \in \mathscr{V}_{\alpha}$ and a $G \in \mathscr{F}$ such that $\left(W_{\alpha, V}^{2}[G]\right)^{\times 2} \subseteq W_{\alpha, U}$. But then $\left(V^{2}\left[p_{\alpha}[G]\right]\right)^{\times 2}=\left(p_{\alpha}\left[W_{\alpha, v}^{2}[G]\right]\right)^{\times 2}$ $\subseteq p_{\alpha}\left[W_{\alpha, U}\right]=U$. Hence $p_{\alpha}[\mathscr{F}]$ is NLU-Cauchy on $\left(X_{\alpha}, \mathscr{V}_{\alpha}\right)$ and therefore converges to some point $x_{\alpha} \in X_{\alpha}$. Consequently, $\mathscr{F}$ itself converges.

(1B) Suppose $X\left\{\left(X_{\alpha}, \mathscr{V}_{\alpha}\right): \alpha \in \Gamma\right\}$ is NLU-complete. $X\left\{\left(X_{\alpha}, \mathscr{V}_{\alpha}\right): \alpha \in \Gamma\right\}$ is a conditions preserving extension of the essentially closed subspace $\iota_{f, \alpha}\left[X_{\alpha}\right]$, so by the previous theorem, $\iota_{f, \alpha}\left[X_{\alpha}\right]$ with the inherited product structure is NLU-complete. Hence so is the isomorphic space $\left(X_{\alpha}, \mathscr{V}_{\alpha}\right)$.

(2A) Suppose each $\left(X_{\alpha}, \mathscr{V}_{\alpha}\right)$ is (strongly) complete. Let $\mathscr{F}$ be a (weak) Cauchy filter on $X\left\{\left(X_{\alpha}, \mathscr{V}_{\alpha}\right): \alpha \in \Gamma\right\}$; then for each $\alpha \in \Gamma$, the image $p_{\alpha}[\mathscr{F}]$ under the projection $p_{\alpha}$ is (weak) Cauchy and hence converges to some point $x_{\alpha}$. Hence $\mathscr{F}$ converges.

(2B) Suppose $\times\left\{\left(X_{\alpha}, \mathscr{V}_{\alpha}\right): \alpha \in \Gamma\right\}$ is strongly complete. Then for each $\beta \in \Gamma$, $\iota_{f, \beta}\left[X_{\beta}\right]$ is an essentially closed subspace of $\times\left\{\left(X_{\alpha}, \mathscr{V}_{\alpha}\right): \alpha \in \Gamma\right\}$ and hence is (strongly) complete. Hence so is $\left(X_{\beta}, \mathscr{V}_{\beta}\right)$.

ACKNOWLEDGEMENTS. I wish to express my gratitude and admiration for T. P. Srinivasan, who supervised my work for this paper, and to whom I owe a major part of my mathematical education. I am also indebted to E. Akin who suggested improvements in proofs and format, to J. Kelley who provided advice and encouragement necessary to survive a "great university," and to the National Science Foundation which provided the money just to survive.

\section{REFERENCES}

1. Manuel P. Berri and R. H. Sorgenfrey, Minimal regular spaces, Proc. Amer. Math. Soc. 14 (1963), 454-458. MR 27 \#2949.

2. Eduard Čech, Topological spaces, Publ. House Czech. Acad. Sci., Prague, 1959; English transl., Interscience, New York, 1966. MR 21 \#2962; MR 35 \#2254.

3. L. W. Cohen, On embedding a space in a complete space, Duke Math. J. 5 (1939), 174-183.

4. A. S. Davis, Indexed systems of neighborhoods for general topological spaces, Amer. Math. Monthly 68 (1961), 886-893. MR 35 \#4869.

5. A. H. Frink, Distance functions and the metrization problems, Bull. Amer. Math. Soc. 43 (1937), 133-142.

6. Paul R. Halmos, Lectures on Boolean algebra, Van Nostrand Math. Studies, no. 1, Van Nostrand, Princeton, N. J., 1963. MR 29 \#4713.

7. E. Hewitt, On two problems of Urysohn, Ann. of Math. (2) 47 (1946), 503-509. MR 8, 165.

8. J. R. Isbell, Uniform spaces, Math. Surveys, no. 12, Amer. Math. Soc., Providence, R. I., 1964. MR 30 \#561.

9. J. L. Kelley, General topology, Van Nostrand, Princeton, N. J., 1955. MR 16, 1136.

10. K. Morita, On the simple extension of a space with respect to a uniformity. I-IV, Proc. Japan. Acad. 27 (1951), 65-72, 130-137, 166-171, 632-636. MR 14, 68; 69; 571.

11. J. Nagata, On a necessary and sufficient condition of metrizability, J. Inst. Polytech. Osaka City Univ. Ser. A Math. 1 (1950), 93-100. MR 13, 264.

12. S. A. Naimpally, Separation axioms in quasi-uniform spaces, Amer. Math. Monthly 74 (1967), 283-284. 
13. V. W. Niemytski, On the third axiom of a metric space, Trans. Amer. Math. Soc. 29 (1927), 507-513.

14. A. H. Stone, Paracompactness and product spaces, Bull. Amer. Math. Soc. 54 (1948), 977-982. MR 10, 204.

15. J. Suzuki, On the metrization and the completion of a space with respect to a uniformity, Proc. Japan. Acad. 27 (1951), 219-223. MR 14, 69.

16. E. Zakon, On uniform spaces with quasi-nested base, Trans. Amer. Math. Soc. 133 (1968), 373-384. MR 37 \#3514.

Department of Mathematics, University of California, Berkeley, California 94720

Current address: Department of Mathematics, Bowling Green State University, Bowling Green, Ohio 43403 